\title{
Onset of Soret driven instability in a Darcy-Maxwell nanofluid
}

\author{
Reema Singh $^{1}\left[\right.$ D Jaimala Bishnoi ${ }^{1} \mathbb{D} \cdot$ Vipin Kumar Tyagi $^{2}$
}

(c) Springer Nature Switzerland AG 2019

\begin{abstract}
This paper presents an analytical and numerical study of the salt particle drift driven by thermal gradient and the nanoparticle drift due to Brownian motion in a Darcy porous layer saturated by a Maxwellian nanofluid under passive management of nanoparticle flux at the boundaries influenced by the Stefan's flow condition. Filtration law of Khuzhayorov et al. (Int J Eng Sci 38:487-504, 2000), for the Darcy-Maxwell fluid, has been used. It is shown that the steady state convection, under both linear and non-linear stability theory do not depend upon the relaxation parameter of the Maxwell fluid. Soret effect is responsible for delaying the stationary convection. It, however, enhances it in comparison to the convection in monodiffusive flow. Oscillatory convection exists, ceases and shifts to the stationary convection. In Soret effected steady convection, among heat, salt and nanoparticles, the transport of nanoparticles is most active while among monodiffusive, double diffusive and Soret governed convection, the heat transfer is most active in Soret induced convection. Streamlines, isotherms and isohalines are shown for steady as well as unsteady convection. Results are compared with some of the existing results.
\end{abstract}

Keywords Darcy-Maxwell nanofluid - Linear and non-linear instability · Passive management of nanoparticle at the boundaries Thermophoresis

Mathematics Subject Classification 76Dxx $\cdot 76 \mathrm{Exx} \cdot 76 \mathrm{Rxx} \cdot 76 \mathrm{Sxx}$

$\begin{array}{llll}\text { List of symbols } & L e & \text { Thermo-solutal Lewis number } \\ c & \text { Nanofluid specific heat at constant pressure } & L n & \text { Thermo-nanofluid Lewis number } \\ C^{*} & \text { Solute concentration }\left(\mathrm{kg} / \mathrm{m}^{3}\right) & N_{A} & \text { Modified diffusivity ratio } \\ C & \text { Dimensionless concentration } & N_{B} & \text { Modified particle-density increment } \\ C_{c}^{*} & \text { Concentration at the upper wall } & N_{C T} & \text { Soret parameter } \\ C_{h}^{*} & \text { Concentration at the lower wall } & p^{*} & \text { Pressure (Pa) } \\ (\rho c)_{m} & \text { Effective heat capacity of the medium }(\mathrm{J} / \mathrm{K}) & p & \text { Dimensionless pressure } \\ (\rho c)_{f} & \text { Effective heat capacity of the fluid }(\mathrm{J} / \mathrm{K}) & R a & \text { Thermal Rayleigh-Darcy number } \\ (\rho c)_{p} & \text { Effective heat capacity of the material con- } & R m & \text { Basic density Rayleigh-Darcy number } \\ & \text { stituting nanoparticles }(\mathrm{J} / \mathrm{K}) & R n & \text { Concentration Rayleigh-Darcy number } \\ d & \text { Dimensional layer depth }(\mathrm{m}) & R s & \text { Solutal Rayleigh number } \\ D_{B} & \text { Brownian diffusion coefficient }\left(\mathrm{m}^{2} / \mathrm{s}\right) & t^{*} & \text { Time (s) } \\ D_{T} & \text { Thermophoretic diffusion coefficient }\left(\mathrm{m}^{2} / \mathrm{s}\right) & t & \text { Dimensionless time } \\ D_{S} & \text { Diffusion coefficient }\left(\mathrm{m}^{2} / \mathrm{s}\right) & T^{*} & \text { Temperature }(\mathrm{K}) \\ \boldsymbol{g} & \text { Gravitational acceleration vector }\left(\mathrm{m} / \mathrm{s}^{2}\right) & T & \text { Dimensionless temperature } \\ K & \text { Permeability of the porous medium }(\mathrm{H} / \mathrm{m}) & T_{c}^{*} & \text { Temperature at the upper wall }\end{array}$

\footnotetext{
$\triangle$ Reema Singh, reemamalik28@gmail.com; Jaimala Bishnoi, jaimalaccsu1@gmail.com; Vipin Kumar Tyagi, prvipin22@gmail.com | ${ }^{1}$ Department of Mathematics, Chaudhary Charan Singh University, Meerut, UP 250004, India. ${ }^{2}$ SBAS, Shobhit Deemed University, Meerut, UP 250110, India.
} 


$\begin{array}{ll}T_{h}^{*} & \text { Temperature at the lower wall } \\ \left(x^{*}, y^{*}, z^{*}\right) & \text { Cartesian coordinates }(\mathrm{m}) \\ (x, y, z) & \text { Dimensionless Cartesian coordinates } \\ \mathbf{v} & \text { Nanofluid velocity }(\mathrm{m} / \mathrm{s}) \\ \mathbf{v}_{\mathbf{D}}^{*} & \text { Darcian velocity }(=\varepsilon \mathbf{v}) \\ \mathbf{v}_{\mathbf{D}} & \text { Dimensionless Darcy velocity } \\ N u & \text { Thermal Nusselt number } \\ N u_{C} & \text { Solutal concentration Nusselt number } \\ N u_{\phi} & \text { Nanoparticle concentration Nusselt number }\end{array}$

\section{Greek symbols}

$\kappa \quad$ Thermal conductivity of the nanofluid (W/ $\mathrm{mK}$ )

$\kappa_{m} \quad$ Effective thermal conductivity of the medium

$\alpha_{m} \quad$ Thermal diffusivity of the medium $\left(\mathrm{m}^{2} / \mathrm{s}\right)$

$\beta_{T} \quad$ Thermal volumetric coefficient $(1 / K)$

$\beta_{C} \quad$ Solutal volumetric coefficient

$\sigma \quad$ Heat capacity ratio

$\varepsilon \quad$ Porosity

$\mu \quad$ Viscosity of the fluid $(\mathrm{kg} / \mathrm{ms})$

$\lambda \quad$ Relaxation time

$\rho \quad$ Fluid density $\left(\mathrm{kg} / \mathrm{m}^{3}\right)$

$\rho_{p} \quad$ Nanoparticle mass density $\left(\mathrm{g} / \mathrm{cm}^{3}\right)$

$\phi^{*} \quad$ Nanoparticle volume fraction $\left(\mathrm{kg} / \mathrm{m}^{3}\right)$

$\phi_{0}^{*} \quad$ Reference value of nanoparticle volume fraction

$\phi \quad$ Dimensionless nanoparticle volume fraction

$\alpha \quad$ Wave number

$\omega \quad$ Frequency of oscillations

$\psi \quad$ Stream function

\section{Subscripts}

$b \quad$ Basic solution

\section{Superscripts}

* Dimensional variable

, Perturbation variable

$$
\begin{array}{ll}
\text { Operators } & \\
\nabla^{2} & \frac{\partial^{2}}{\partial x^{2}}+\frac{\partial^{2}}{\partial y^{2}}+\frac{\partial^{2}}{\partial z^{2}} \\
\nabla_{1}^{2} & \frac{\partial^{2}}{\partial x^{2}}+\frac{\partial^{2}}{\partial z^{2}}
\end{array}
$$

\section{Introduction}

In 1879, the Swiss scientist Soret [2] performed an experiment with sodium chloride and potassium nitrate in a tube by maintaining the top and bottom ends of it at $800^{\circ} \mathrm{C}$ and room temperature respectively. He noticed the non-uniformity in the concentration of salt and the migration of colloidal particles to the cold end and the hot end of the tube. He concluded that in isotropic fluid systems, in the absence of external forces, concentration gradient is induced by the driving force of applied temperature gradient until the system reaches into the steady state condition. During 1879 to 1884 , Soret quantified the effect for many electrolyte solutions which were later summarized and discussed by Costesèque [3]. Although, Ludwig [4] observed and presented the phenomena several years before Soret in his brief lecture titled 'On Haze and Dust' in Royal Institute and later incorporated it in the book 'Scientific Addresses', but he was overlooked and the phenomenon was named as Soret effect. Later, it was also called Ludwig-Soret effect or thermodiffusion or thermal diffusion. The same phenomenon of suspended particles in carrier liquid or gas is called thermophoresis. Thus thermophoresis is the transport mechanism responsible for the formation of concentration currents. In 1962, De Groot and Mazur [5] appreciated this phenomenon and formulated it in the book 'Non-Equilibrium Thermodynamics'. Considering the linear laws of irreversible thermodynamics, the mass flux $\mathbf{J}_{\mathbf{i}}$ of the ith component in a multi-component mixtures (say $p$ components), neglecting pressure gradient, is written as

$\mathbf{J}_{\mathbf{i}}=-\rho_{0} \sum_{k=1}^{p-1} D_{i k} \nabla C_{i}-\rho_{0} D_{T i} C_{i}\left(1-C_{i}\right) \nabla T$

where $\rho_{0}$ is the specific mass or density, $D_{i k}$ is the isothermal molecular concentration diffusivity, $D_{T i}$ is the thermodiffusion coefficient, $C_{i}$ is the concentration of the reference component, $\nabla C_{i}$ is the gradient of reference concentration induced by temperature gradient $\nabla T$.

Thus, the conservation equation for concentration field is written as

$\left(\frac{\partial}{\partial t}+q \cdot \nabla\right) C_{i}=-\nabla \cdot \mathbf{J}_{\mathbf{i}^{\prime}}$

where $q$ represents the velocity field. Soret effect is characterized by Soret coefficient $S_{T}$, the ratio of the thermal diffusion coefficient to the molecular diffusion coefficient. It is proportional to the volume fraction generally having the magnitude in the range of $10^{-3}-10^{-2} \mathrm{~K}^{-1}$ for gases or simple electrolytes [6] but can be of larger magnitudes for macromolecular and colloidal solutions. Soret coefficient may be positive or negative depending on the sense of migration of the reference components. Positive Soret coefficient means the denser colloids are forced to move towards lower temperature regions (regions of smaller agitation), while negative Soret coefficient means the accumulation of particles towards the regions of higher temperatures. In a simple fluid heated from below, viscous friction and thermal conductivity hamper the convection 
resulting in local density fluctuations, known as Rayleigh-Bènard convection while in mixtures, thermodiffusion, positive or negative (in case of fluid layer heated from below or above, see [7] for negative thermodiffusion), sets inverted concentration gradients that can only be relaxed by mass diffusion. Since it is far slower than heat transport, the convection threshold is substantially lowered. Both the situations are very commonly observed but no study has predicted the direction of thermodiffusion motion for a given solute. An overview on the basic physics of the Soret effect has been explained by Köhler and Wiegand [8]. The mechanism of thermophoresis works for nanoparticles as well. Thus, the Soret coefficient may be different for different matters of concentration. The mechanism of thermophoresis works for nanoparticles as well in such a way that the Soret coefficient may be different for different matters of concentration.

Since its discovery, large number of theoretical and experimental studies have established its importance as an agent of various convective phenomena like multiple steady oscillatory states, subcritical flows, standing-traveling waves, Hopf bifurcations and hysteresis effects (see [9-12] and references therein).

In a convective configuration, thermophoresis has a number of applications in many naturally occurring processes. Whether it is the operation of solar ponds [13] or the thermohaline convection in oceans [14] or the thermodiffusion in stars [15], convection is largely affected by thermophoresis. It is also helpful in the prediction of the concentrations of the different components of hydrocarbon mixtures of earth's hydrocarbon reservoirs which is necessary for the optimum oil/gas recovery $[16,17]$. Soret diffusion might also serve as an agent to fractionate isotopes of the elements like $\mathrm{Mg}, \mathrm{Ca}, \mathrm{Fe}, \mathrm{Si}, \mathrm{O}, \mathrm{Sr}, \mathrm{Hf}$, and $\mathrm{U}$ found in high-temperature geochemical melts. Fractionation of isotopes is useful in a wide range of natural condensed systems, including the early solar system and the atmospheric water cycle. It is also helpful in mapping the temperature gradient in magma chambers, and in defining the initial isotopic composition of the earth from the solar nebula [18].

In biological systems, the mass transport across biological membranes of an organ or a tumor is induced by small thermal gradients and in some cases even the shape of the membrane may be manipulated by them $[19,20]$.

Thermophoresis helps in measuring bimolecular affinities in complex liquids and provides the basis for a size selective molecule trap. It has also been exploited as an industrial tool for aerosol collectors (thermal precipitators), gas cleaning, nuclear reactor safety, and the corrosion/ fouling of heat exchangers. Paintings and other works of art may have a protection shield against airborne particles through thermophoresis [21]. Measurement of Soret coefficient is also important to conduct material science experiments and to measure their physical properties in a low-gravity environment, i.e. crystal growth from solution or vapor phase deposition on Skylab or space shuttle missions. For some of the useful experimental and theoretical studies predicting a noticeable Soret effect, the work of Hardin et al. [10] and references therein are worth mentioning. Recently many of the fields mentioned so far are concerned with nanofluids. Nanofluids are more stable suspensions having acceptable viscosity, better wetting capacity, and dispersion properties. In nanofluids the concentration flux of nanoparticles is also induced by thermal gradient resulting in a thermophoresis of nanoparticles. Recently Derakhshan et al. [22], Zangooee [23], Ghadikolaei et al. [24-27], Gholinia et al. [28], Hosseinzadeh et al. $[29,30]$ have studied convection in some important nanofluids considering the thermophoresis of nanoparticles. When a pure base fluid in a nanofluid is replaced by a binary fluid, the convection becomes more interesting. The simultaneous occurrence of two types of thermophoresis, the one induced by the thermal gradient affecting the flux of salt and the other the thermophoresis of nanoparticles, make binary nanofluids applicable in variety of natural fields.

There are a number of nanofluids exhibiting the exceptional behavior regarding viscosity and other properties of non-Newtonian medium but unfortunately, only a very few theoretical studies on Soret-driven thermosolutal instability in non-Newtonian nanofluids are reported in literature.

The main purpose of the present work is to explore the role of Soret effect on the thermal instability of a binary nanofluid when Bénard convection is governed by the Darcy Maxwell model, introduced by Alishayev [31]. Khuzhayorov et al. [1] interpreted the notion of macroscopic filtration law for describing transient linear viscoelastic fluid flows in porous media. The model has been used by Wang and Tan [32] to discuss the effect of Soret parameter on a regular Maxwell fluid. There are some other studies where the stability of single phase Maxwell fluid have been investigated using this model [33-38]. Recently, Jaimala et al. [39] used the model to investigate the classical Horton Rogers Lapwood problem for thermal diffusion in nanofluid. The important aspect to mention is that the present investigation deals with the Stefan's flow condition which says that in natural situations the mass flux of fluid can only be controlled passively at the boundaries (see Baehr and Stephan [40]). Adopting the Stefan's condition for the flux of nanoparticles at the boundaries, it is supposed that there is zero flux of nanoparticles. Nield and Kuznetsov [41] followed Stefan's conditions to discuss the thermal stability of Newtonian nanofluid. Jaimala et al. [39] used this condition in discussing the thermal stability of Darcy-Maxwell nanofluid and showed that 
the nanoparticles are accountable for reduction in Horton-Rogers Rayleigh number notably. This paper reveals that the presence of nanoparticles in combination of weak concentration diffusivity and the pronounced solutal buoyancy force significantly alter the classical Horton-Rogers-Lapwood convection as compared to regular Maxwell fluid [42].

\section{Formulation}

The proposed model deals with the binary nanofluid suspension under the following assumptions:

a. Binary nanofluid is a dilute mixture, i.e. $\phi \ll 1$. This assumption is consistent with almost every nanofluid studied so far.

b. Nanoparticles and the binary fluid are in local-thermalequilibrium (LTE). This situation is coherent with the size of nanoparticles such that the internal equilibration times are of the order of $1 \mathrm{~ns}$, which is negligible as compared with the fluid-dynamics time scales.

c. Chemical reactions are negligible.

d. External forces except gravitational force, radiative heat transfer and viscous dissipation are negligible.

e. The fluid layer is heated and soluted from below.

f. The soluted nanofluid flow is subjected to thermophoresis of salt and thermophoresis of nanoparticles.

g. Particle-related effects like particle inertia, Magnus effects etc. have not been considered.

h. Darcy Maxwellian fluid model is designed by the filtration law proposed by Khuzhayorov et al. [1].

i. Analogous to Stefan's flow there is no nanoparticle flux at the boundaries.

The conservation equation for mass is taken as

$\nabla^{*} \cdot \mathbf{v}_{D}^{*}=0$,

Following Khuzhayorov et al. [1], the momentum equation can be written as

$\frac{\mu}{K} \mathbf{v}_{D}^{*}=\left(1+\lambda^{*} \frac{\partial}{\partial t^{*}}\right)\left(-\nabla^{*} p^{*}+\rho \mathbf{g}\right)$.

Following Eastman et al. [43], the density of the salted nanofluid can be computed as the volume weighted average. Therefore, using the volume fractions $\phi$ as weights, density can be written as

$\rho=\left[\phi^{*} \rho_{p}+\left(1-\phi^{*}\right)\left\langle\rho\left\{1-\beta_{T}\left(T^{*}-T_{c}^{*}\right)-\beta_{C}\left(C^{*}-C_{c}^{*}\right)\right\}\right\rangle\right]$,

where $\rho\left\{1-\beta_{T}\left(T^{*}-T_{c}^{*}\right)-\beta_{C}\left(C^{*}-C_{c}^{*}\right)\right\}$ is the density of the soluted base fluid.

Thus using (5), Eq. (4) becomes

$$
\begin{aligned}
\frac{\mu}{K} \mathbf{v}_{D}^{*}=\left(1+\lambda^{*} \frac{\partial}{\partial t^{*}}\right)\left[-\nabla^{*} p^{*}+\left(\phi^{*} \rho_{p}+\left(1-\phi^{*}\right)\right.\right. \\
\left.\left.\left\langle\rho\left\{1-\beta_{T}\left(T^{*}-T_{c}^{*}\right)-\beta_{C}\left(C^{*}-C_{c}^{*}\right)\right\}\right\rangle\right) \mathbf{g}\right],
\end{aligned}
$$

Following Buongiorno [44] and Nield and Kuznetsov [45], equation of conservation of thermal energy is written as

$$
\begin{aligned}
& (\rho c)_{m} \frac{\partial T^{*}}{\partial t^{*}}+(\rho c)_{f} \mathbf{v}_{D}^{*} \cdot \nabla^{*} T^{*} \\
& =\kappa_{m} \nabla^{* 2} T^{*}+\varepsilon(\rho c)_{p}\left[D_{B} \nabla^{*} \phi^{*} \cdot \nabla^{*} T^{*}+\left(D_{T} / T_{c}^{*}\right) \nabla^{*} T^{*} \cdot \nabla^{*} T^{*}\right] .
\end{aligned}
$$

Following Hurle et al. [46], it is assumed that the value of mass fraction is very small (i.e. $c_{0} \ll 1$ ), therefore, for a fluid saturated in a porous medium, having two types of concentrations, salt and nanoparticles, Eqs. (2) using (1) can be written for the two species as

$\frac{\partial C^{*}}{\partial t^{*}}+\frac{1}{\varepsilon} \mathbf{v}_{D}^{*} \cdot \nabla^{*} C^{*}=D_{S} \nabla^{* 2} C^{*}+D_{C T} \nabla^{* 2} T^{*}$,

$\frac{\partial \phi^{*}}{\partial t^{*}}+\frac{1}{\varepsilon} \mathbf{v}_{D}^{*} \cdot \nabla^{*} \phi^{*}=D_{B} \nabla^{* 2} \phi^{*}+\left(D_{T} / T_{c}^{*}\right) \nabla^{* 2} T^{*}$,

where $D_{S}$ is the diffusion coefficient of salt, $D_{C T}$ is the Soret coefficient of salt, $D_{B}$ is the Brownian diffusion coefficient and $D_{T}$ is the Soret coefficient of nanoparticles.

Let an infinite horizontal fluid layer of depth $d$ be saturated in a porous layer lies between two stress free, rigid, perfectly conducting and impervious boundaries situated at $z=0$ and $z=d$. The layer is heated and salted from below and the thermal and salinity gradients are constant (Fig. 1). At $z=0$ and $z=d$, the thermal and salt concentrations are constant and the flux of nanoparticle concentration is zero there (Stefan's flow) such that the associated boundary conditions are

$\mathbf{v}_{D}^{*}=0, T^{*}=T_{h^{\prime}}^{*}, C^{*}=C_{h^{\prime}}^{*} D_{B} \frac{\partial \phi^{*}}{\partial z^{*}}+\frac{D_{T}}{T_{c}^{*}} \frac{\partial T^{*}}{\partial z^{*}}=0$ at $z=0$,

$\mathbf{v}_{D}^{*}=0, T^{*}=T_{c^{\prime}}^{*}, C^{*}=C_{c^{\prime}}^{*} D_{B} \frac{\partial \phi^{*}}{\partial z^{*}}+\frac{D_{T}}{T_{c}^{*}} \frac{\partial T^{*}}{\partial z^{*}}=0$ at $z=d$.

Choosing $d, \frac{\sigma d^{2}}{\alpha_{m}}, \frac{\alpha_{m}}{d}, \frac{\alpha_{m}}{d^{2}}$ and $\frac{\mu \alpha_{m}}{K}$ as the units of length, time, velocity, relaxation time and pressure, where $\alpha_{m}=\frac{\kappa_{m}}{(\rho c)_{f}}$ and $\sigma=\left(\frac{(\rho c)_{m}}{(\rho c)_{f}}\right)$, and assuming

$\phi=\frac{\phi^{*}-\phi_{0}^{*}}{\phi_{0}^{*}}, \quad T=\frac{T^{*}-T_{c}^{*}}{T_{h}^{*}-T_{c}^{*}}, C=\frac{C^{*}-C_{c}^{*}}{C_{h}^{*}-C_{c}^{*}}$,

Equations (3), (6) to (9) and the boundary conditions (10) and (11) are rendered dimensionless and we obtain 
Fig. 1 Physical configuration of the problem

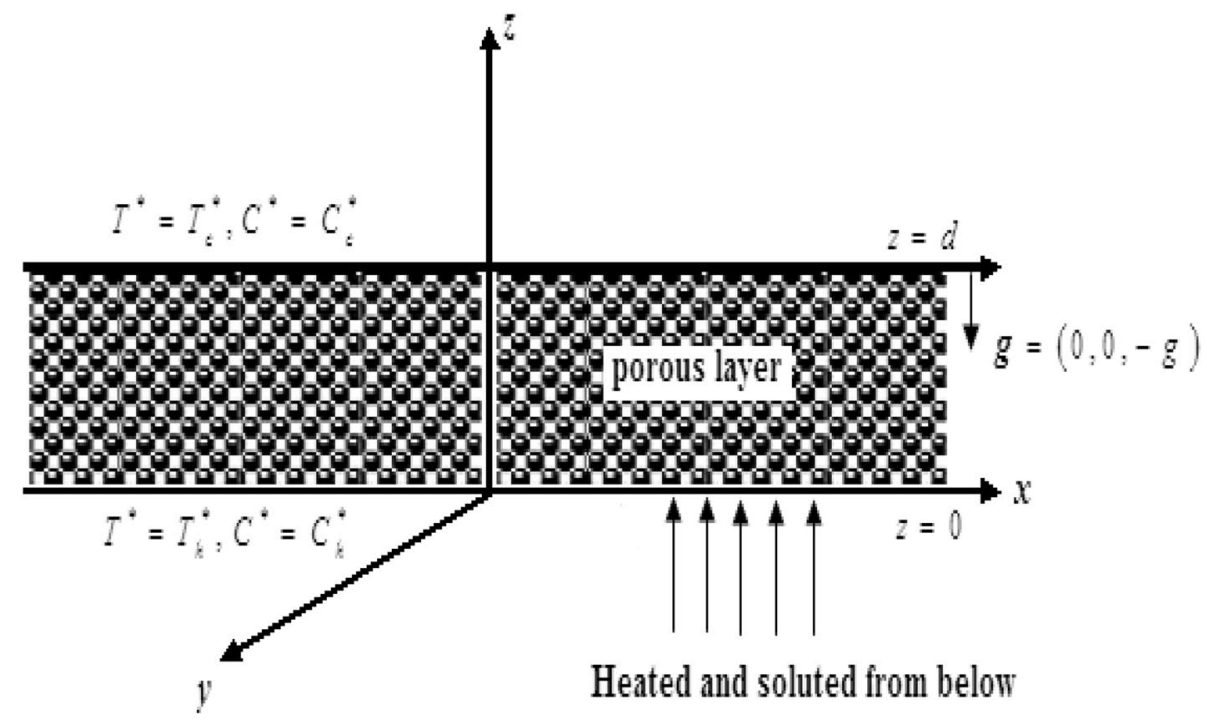

$\nabla \cdot \mathbf{v}=0$

$\mathbf{v}=\left(1+\frac{\lambda}{\sigma} \frac{\partial}{\partial t}\right)\left(-\nabla p-R m \hat{\mathbf{e}}_{\mathbf{z}}+\operatorname{RaT} \hat{\mathbf{e}}_{\mathbf{z}}-\operatorname{Rn\phi } \hat{\mathbf{e}}_{\mathbf{z}}+\frac{R s}{L n} C \hat{\mathbf{e}}_{\mathbf{z}}\right)$,

$\frac{\partial T}{\partial t}+\mathbf{v} \cdot \nabla T=\nabla^{2} T+\frac{N_{B}}{L e} \nabla \phi \cdot \nabla T+\frac{N_{A} N_{B}}{L e} \nabla T \cdot \nabla T$,

$\frac{1}{\sigma} \frac{\partial C}{\partial t}+\frac{1}{\varepsilon} \mathbf{v} \cdot \nabla C=\frac{1}{L n} \nabla^{2} C+N_{C T} \nabla^{2} T$

$\frac{1}{\sigma} \frac{\partial \phi}{\partial t}+\frac{1}{\varepsilon} \mathbf{v} \cdot \nabla \phi=\frac{1}{L e} \nabla^{2} \phi+\frac{N_{A}}{L e} \nabla^{2} T$,

$\mathbf{v}=0, T=1, C=1, \frac{\partial \phi}{\partial z}+N_{A} \frac{\partial T}{\partial z}=0$ at $z=0$,

$\mathbf{v}=0, T=0, C=0, \frac{\partial \phi}{\partial z}+N_{A} \frac{\partial T}{\partial z}=0$ at $z=1$.

Where $\nabla^{2} \equiv \frac{\partial^{2}}{\partial x^{2}}+\frac{\partial^{2}}{\partial y^{2}}+\frac{\partial^{2}}{\partial z^{2}}$

$R m=\frac{\left[\rho_{p} \phi_{0}^{*}+\rho\left(1-\phi_{0}^{*}\right)\right] g K d}{\mu \alpha_{m}}$ is the "basic density RayleighDarcy number",

$R a=\frac{\rho g \beta K d\left(T_{h}^{*}-T_{c}^{*}\right)}{\mu \alpha_{m}}$ is the "thermal Rayleigh-Darcy number",

$R n=\frac{\left(\rho_{p}-\rho\right) \phi_{0}^{*} g K d}{\mu \alpha_{m}}$ is the "concentration Rayleigh-Darcy number",

$R s=\frac{\rho g K d \beta_{c}\left(C_{h}^{*}-C_{c}^{*}\right)}{\mu D_{s}}$ is the "solutal Rayleigh-Darcy number",

$N_{A}=\frac{D_{T}\left(T_{h}^{*}-T_{c}^{*}\right)}{D_{B} T_{c}^{*} \phi_{0}^{*}}$ is the "modified diffusivity ratio",
$N_{B}=\frac{\varepsilon \phi_{0}^{*}(\rho c)_{p}}{(\rho c)_{f}}$ is the "modified particle-density increment",

$L e=\frac{\alpha_{m}}{D_{B}}$ is "thermo-nanofluid Lewis number",

$L n=\frac{\alpha_{m}^{B}}{D_{0}}$ is the "thermo-solutal Lewis number",

$N_{C T}=\frac{D_{\Phi_{C T}\left(T_{h}^{*}-T_{c}^{*}\right)}}{\left(C_{h}^{*}-C_{c}^{*}\right)}$ is the "Soret parameter".

$\mathbf{v}_{\text {D }}$ is replaced by $\mathbf{v}$ for convenience.

We follow a time-independent stagnant solution of Eqs. (12)-(16) such that temperature, concentration and nanoparticle volume fraction vary in z-direction only. Thus, the basic state is described by:

$\mathbf{v}=0, p=p_{b}(z), T=T_{b}(z), C=C_{b}(z), \phi=\phi_{b}(z)$,

$\frac{d^{2} T_{b}}{d z^{2}}+\frac{N_{B}}{L e} \frac{d \phi_{b}}{d z} \frac{d T_{b}}{d z}+\frac{N_{A} N_{B}}{L e}\left(\frac{d T_{b}}{d z}\right)^{2}=0$,

$\frac{d^{2} C}{d z^{2}}=0$

$\frac{d^{2} \phi_{b}}{d z^{2}}+N_{A} \frac{d^{2} T_{b}}{d z^{2}}=0$

It provides the steady state solutions as

$T_{b}=1-z$,

$C_{b}=1-z$

$\phi_{b}=\phi_{0}+N_{A} z$

\subsection{Linear stability analysis}

Let the initial steady state be slightly perturbed. Following the classical lines of linear stability theory presented by 
Chandrasekhar [47], the perturbations are decomposed in terms of normal modes in the form

$\left(w^{\prime}, T^{\prime}, C^{\prime}, \phi^{\prime}\right)=[W(z), \Theta(z), \eta(z), \Phi(z)] \exp (s t+i l x+i m y)$,

where I (in $x$-direction) and $m$ (in $y$-direction) are dimensionless wave numbers respectively and $s\left(=\omega_{r}+i \omega_{i}\right)$ is a complex number predicting about the perturbations. So we arrive at the following boundary value problem:

$\left(D^{2}-\alpha^{2}\right) W+\alpha^{2}\left(1+\frac{\lambda s}{\sigma}\right)\left(R a \Theta-R n \Phi+\frac{R s}{L n} \eta\right)=0$,

$W+\left(D^{2}-\alpha^{2}-\frac{N_{A} N_{B}}{L e} D-s\right) \Theta-\frac{N_{B}}{L e} D \Phi=0$,

$\frac{W}{\varepsilon}+N_{C T}\left(D^{2}-\alpha^{2}\right) \Theta+\left(\frac{1}{L n}\left(D^{2}-\alpha^{2}\right)-\frac{s}{\sigma}\right) \eta=0$,

$N_{A} \frac{W}{\varepsilon}-\frac{N_{A}}{L e}\left(D^{2}-\alpha^{2}\right) \Theta-\left(\frac{1}{L e}\left(D^{2}-\alpha^{2}\right)-\frac{s}{\sigma}\right) \Phi=0$,

$W=0, \Theta=0, \eta=0, D \Phi+N_{A} D \Theta=0$ at $z=0$ and $z=1$,

where $D \equiv \frac{d}{d z}$ and $\alpha=\left(I^{2}+m^{2}\right)^{1 / 2}$ is a dimensionless horizontal wave number.

Here it is to be noted that the boundary value problem for Darcy-Maxwell nanofluid converts to Horton-Roger-Lapwood problem [42] for a double diffusive convection in a regular nanofluid as soon as the relaxation parameter $\lambda \rightarrow 0$.

To obtain an approximate solution for the system of Eqs. (27)-(31), we use the Galerkin-type-weighted residual method. Choosing trial functions (satisfying the boundary conditions) $W_{p^{\prime}} \Theta_{p^{\prime}} \Phi_{p}$ and $\eta_{p}: p=1,2,3 \ldots N$ and writing

$W=\sum_{p=1}^{N} A_{p} W_{p^{\prime}} \Theta=\sum_{p=1}^{N} B_{p} \Theta_{p^{\prime}} \Phi=\sum_{p=1}^{N} C_{p} \Phi_{p^{\prime}} \eta=\sum_{p=1}^{N} D_{p} \eta_{p^{\prime}}$

we obtain a system of $4 N$ linear algebraic equations in the $4 N$ unknowns $A_{p}, B_{p}, C_{p}$ and $D_{p}$. In view of the boundary conditions given by Eq. (31), the base functions mentioned in (32) may be assumed as

$W_{p}=\Theta_{p}=\eta_{p}=\sin p \pi z ; \Phi_{p}=-N_{A} \sin p \pi z$.

Substituting Eqs. (33) in (32) and using the results in Eqs. (27)-(31), we get a system of equations of non-trivial solution for the first approximation i.e. at $\mathrm{N}=1$, under the necessary condition

$\left|\begin{array}{cccc}-\delta^{2} & \alpha^{2}\left(1+\frac{s \lambda}{\sigma}\right) R a & \alpha^{2}\left(1+\frac{s \lambda}{\sigma}\right) \frac{R s}{L n} & \alpha^{2}\left(1+\frac{s \lambda}{\sigma}\right) R n N_{A} \\ 1 & -\left(\delta^{2}+s\right) & 0 & 0 \\ \frac{1}{\varepsilon} & -\delta^{2} N_{C T} & -\left(\frac{\delta^{2}}{L n}+\frac{s}{\sigma}\right) & 0 \\ \frac{1}{\varepsilon} & \frac{\delta^{2}}{L e} & 0 & -\left(\frac{\delta^{2}}{L e}+\frac{s}{\sigma}\right)\end{array}\right|=0$, where $\delta^{2}=\pi^{2}+\alpha^{2}$.

\subsubsection{Stationary convection}

It follows from Eq. (34) that the stationary convection at the marginal state $(s=0)$ is governed by the Rayleigh number

$R a^{s t}=\frac{\delta^{4}}{\alpha^{2}}-R n N_{A}\left(1+\frac{L e}{\varepsilon}\right)-\frac{R s}{\varepsilon}\left(1-\varepsilon N_{C T}\right)$,

Such that the critical Rayleigh number $R a^{\text {st }}$ at the critical wave number $\alpha=\pi$ is obtained as

$R a^{s t}=4 \pi^{2}-\left(1+\frac{L e}{\varepsilon}\right) N_{A} R n-\left(1-\varepsilon N_{C T}\right) \frac{R s}{\varepsilon}$,

In the absence of Soret parameter $\left(N_{C T}=0\right)$, Eq. (36) converts to

$R a^{s t}=4 \pi^{2}-R n N_{A}\left(1+\frac{L e}{\varepsilon}\right)-\frac{R s}{\varepsilon}$,

which is obtained by Jaimala et al. [48].

Equation (37) in the absence of salt, converts to

$R a^{s t}=4 \pi^{2}-R n N_{A}\left(1+\frac{L e}{\varepsilon}\right)$.

This is the Rayleigh number obtained by Jaimala et al. [39].

\subsubsection{Oscillatory convection}

When $\left(s=\omega_{i}+i \omega_{r}: \omega_{r}=0\right)$ at the marginal state, the Rayleigh number is obtained as:

$R a^{\text {osc }}=\frac{\sigma}{\alpha^{2} \varepsilon}\left[\frac{\left(X_{1}-X_{2}+X_{3}\right) U+\omega^{2}\left(Y_{1}-Y_{2}+Y_{3}\right) V}{U^{2}+V^{2}}\right]$,

where

$X_{1}=\alpha^{2} R n N_{A}\left[\delta^{4} \sigma^{2}(\varepsilon+L e)-\lambda \delta^{2} \omega^{2}(\sigma L e+\varepsilon L n+L n L e)-\omega^{2} \sigma L e L n\right]$,

$X_{2}=\delta^{4}\left[\delta^{4} \sigma^{2}-\omega^{2}\{L n \sigma+L e \sigma+L e L n\}\right]$,

$X_{3}=\alpha^{2} R s\left[\delta^{4} \sigma^{2}\left(1-N_{C T} \varepsilon\right)-\omega^{2}\left\{\delta^{2} \lambda \sigma+L e\left(\sigma-\delta^{2} \lambda \varepsilon N_{C T}+\delta^{2} \lambda\right)\right\}\right]$,

$Y_{1}=\alpha^{2} R n N_{A}\left[\sigma \delta^{2}\left\{\lambda \delta^{2}(\varepsilon+L e)+(\sigma L e+\varepsilon L n+L e L n)\right\}-\lambda \omega^{2} L e L n\right]$,

$Y_{2}=\varepsilon \delta^{2}\left[\delta^{4} \sigma(L e+\sigma+L n)-\omega^{2} L e L n\right]$,

$Y_{3}=\alpha^{2} R s\left\{\delta^{2} \sigma\left(L e-\varepsilon N_{C T} L e+\sigma-\lambda \delta^{2} \varepsilon N_{C T}+\lambda \delta^{2}\right)-\lambda \omega^{2} L e\right\}$,

$U=\left[\omega^{2} \sigma\left\{\lambda \delta^{2}(L e+L n)+L e L n\right\}-\delta^{4} \sigma^{3}\right]$,

$V=\left[\lambda \omega^{2} \operatorname{LnLe}-\delta^{2} \sigma^{2}\left(\lambda \delta^{2}+\operatorname{Ln}+L e\right)\right]$.

We write $\omega$ in place of $\omega_{i}$ for convenience.

The frequency of oscillation is given by the following fourth order equation in $\omega$ : 


$$
\begin{gathered}
\left(P_{1} P_{2}+P_{3} P_{4}\right) \omega^{4}+\left(P_{1} P_{5}+P_{2} P_{6}+P_{3} P_{7}\right. \\
\left.\quad+P_{4} P_{8}\right) \omega^{2}+\left(P_{5} P_{6}+P_{7} P_{8}\right)=0
\end{gathered}
$$

where
$C=D_{11}(t) \cos (\alpha x) \sin (\pi z)+D_{02}(t) \sin (2 \pi z)$,

where the coefficients $A_{11}(t), B_{11}(t), B_{02}(t), C_{11}(t), C_{02}(t)$, $D_{11}(t)$ and $D_{02}(t)$ are time dependent amplitudes and are

$$
\begin{aligned}
& P_{1}=\delta^{4} \varepsilon(\sigma L n+\sigma L e+L e L n)-\alpha^{2} R n N_{A}\left\{\sigma L e L n+\lambda \delta^{2}(\sigma L e+\varepsilon L n+L e L n)\right\} \\
& -\alpha^{2} R s\left\{\delta^{2} \lambda\left(L e+\sigma-\operatorname{LeN}_{C T} \varepsilon\right)+\sigma L e\right\}, \\
& P_{2}=-\lambda L e L n, \\
& P_{3}=L e\left(\delta^{2} \varepsilon L n-\alpha^{2} \lambda R n N_{A} L n-\lambda \alpha^{2} R s\right), \\
& P_{4}=\sigma\left\{\delta^{2} \lambda(L e+L n)+L e L n\right\}, \\
& P_{5}=\delta^{2} \sigma^{2}\left(\lambda \delta^{2}+L n+L e\right) \text {, } \\
& P_{6}=\delta^{4} \sigma^{2}\left\{\alpha^{2} R n N_{A}(\varepsilon+L e)-\delta^{4} \varepsilon+\alpha^{2} R s\left(1-N_{C T} \varepsilon\right)\right\} \text {, } \\
& P_{7}=-\delta^{4} \sigma^{3} \text {, } \\
& P_{8}=\delta^{2} \sigma\left[\begin{array}{c}
\alpha^{2} R n N_{A}\left\{\delta^{2} \lambda(\varepsilon+L e)+\sigma L e+\varepsilon L n+L e L n\right\}-\delta^{4} \varepsilon(\sigma+L e+L n) \\
+\alpha^{2} R s\left\{\left(1-N_{C T} \varepsilon\right)\left(L e+\delta^{2} \lambda\right)+\sigma\right\}
\end{array}\right] .
\end{aligned}
$$

\subsection{Nonlinear stability analysis}

To get the information about the amplitude of motion and the behaviour of heat, salt and mass transfer, non-linear stability theory is used. After substituting $T_{b}, C_{b}$ and $\phi_{b}$ given by Eqs. (23), (24) and (25) respectively in the twodimensional perturbed state solution, after elimination of pressure and introduce the stream function in the resulting equation, we get

$\nabla_{1}^{2} \psi=\left(1+\frac{\lambda}{\sigma} \frac{\partial}{\partial t}\right)\left[-R a \frac{\partial T}{\partial x}+R n \frac{\partial \phi}{\partial x}-\frac{R s}{L n} \frac{\partial C}{\partial x}\right]$,

$\frac{\partial T}{\partial t}+\frac{\partial \psi}{\partial x}=\nabla_{1}^{2} T+\frac{\partial(\psi, T)}{\partial(x, z)}$

$\frac{1}{\sigma} \frac{\partial C}{\partial t}+\frac{1}{\varepsilon}\left(\frac{\partial \psi}{\partial x}\right)=\frac{1}{L n} \nabla_{1}^{2} C+\frac{1}{\varepsilon} \frac{\partial(\psi, C)}{\partial(x, z)}+N_{C T} \nabla_{1}^{2} T$

$\frac{1}{\sigma} \frac{\partial \phi}{\partial t}-\frac{N_{A}}{\varepsilon}\left(\frac{\partial \psi}{\partial x}\right)=\frac{1}{L e} \nabla_{1}^{2} \phi+\frac{N_{A}}{L e} \nabla_{1}^{2} T+\frac{1}{\varepsilon} \frac{\partial(\psi, \phi)}{\partial(x, z)}$,

where $\nabla_{1}^{2}=\frac{\partial^{2}}{\partial x^{2}}+\frac{\partial^{2}}{\partial z^{2}}$.

Now Eq. (31) converts into

$\psi=\frac{\partial^{2} \psi}{\partial z^{2}}=T=0, C=0, \frac{\partial \phi}{\partial z}+N_{A} \frac{\partial T}{\partial z}=0$ at $z=0$ and $z=1$.

We take Fourier expressions for $\psi, T, \phi$ and $C$ to perform nonlinear stability analysis as

$\psi=A_{11}(t) \sin (\alpha x) \sin (\pi z)$,

$T=B_{11}(t) \cos (\alpha x) \sin (\pi z)+B_{02}(t) \sin (2 \pi z)$,

$\phi=-N_{A} C_{11}(t) \cos (\alpha x) \sin (\pi z)-N_{A} C_{02}(t) \sin (2 \pi z)$, to be determined from the dynamics of the system. Substituting Eqs. (46)-(49) into the set of coupled non-linear partial differential equations given by (41)-(44) and applying the orthogonality condition in corresponding eigenfunctions, we have

$$
\begin{aligned}
A_{11}=- & \frac{\alpha}{\delta^{2}}\left\{R a B_{11}+R n N_{A} C_{11}+\frac{R s}{L n} D_{11}+\frac{\lambda}{\sigma}\right. \\
& \left.\left(R a \frac{d B_{11}}{d t}+R n N_{A} \frac{d C_{11}}{d t}+\frac{R s}{L n} \frac{d D_{11}}{d t}\right)\right\},
\end{aligned}
$$

$\frac{d B_{11}}{d t}=-\left(\alpha A_{11}+\delta^{2} B_{11}+\pi \alpha A_{11} B_{02}\right)$,

$\frac{d B_{02}}{d t}=\frac{1}{2}\left(\pi \alpha A_{11} B_{11}-8 \pi^{2} B_{02}\right)$,

$\frac{d C_{11}}{d t}=-\sigma\left\{\frac{\alpha}{\varepsilon} A_{11}-\frac{\delta^{2}}{L e}\left(B_{11}-C_{11}\right)+\frac{\pi \alpha}{\varepsilon} A_{11} C_{02}\right\}$

$\frac{d C_{02}}{d t}=\frac{\sigma}{2}\left\{\frac{\pi \alpha}{\varepsilon} A_{11} C_{11}+\frac{8 \pi^{2}}{L e}\left(B_{02}-C_{02}\right)\right\}$,

$\frac{d D_{11}}{d t}=-\sigma\left[\frac{\alpha}{\varepsilon} A_{11}+\delta^{2} N_{C T} B_{11}+\frac{\delta^{2}}{L n} D_{11}+\frac{\pi \alpha}{\varepsilon} A_{11} D_{02}\right]$,

$\frac{d D_{02}}{d t}=\frac{\sigma}{2}\left[\frac{\pi \alpha}{\varepsilon} A_{11} D_{11}-8 \pi^{2}\left(\frac{D_{02}}{L n}+B_{02} N_{C T}\right)\right]$.

Equations (50)-(56) represent a non-linear autonomous system of simultaneous ordinary differential equations, which is not suitable to analytical analysis. Therefore, it is to be solved numerically using Runge-Kutta-Gill 
method. For steady state, setting the left-hand sides of Eqs. (51)-(56) equal to zero, the resulting equations are solved and the amplitudes are obtained in terms of $A_{11}$ as:

$B_{11}=-\frac{\alpha A_{11}}{\delta^{2}+\alpha^{2}\left(A_{11}^{2} / 8\right)}$

$B_{02}=-\frac{\alpha^{2}\left(A_{11}^{2} / 8\right)}{\pi\left(\delta^{2}+\alpha^{2}\left(A_{11}^{2} / 8\right)\right)}$,

$$
\begin{aligned}
N u(t) & =\frac{\text { Heat transport by (conduction }+ \text { convection) }}{\text { Heat transport by conduction }} \\
& =1+\left[\frac{\int_{0}^{2 \pi / \alpha_{c}}\left(\frac{\partial T}{\partial z}\right) d x}{2 \pi / \alpha_{c}}\left(\frac{\partial T_{b}}{\partial z}\right) d x\right]_{z=0},
\end{aligned}
$$

provides

$N u(t)=1-2 \pi B_{02}(t)$,

The use of Eqs. (57)-(62) into (50) leads to

$P\left(A_{11}^{2} / 8\right)^{3}+Q\left(A_{11}^{2} / 8\right)^{2}+R\left(A_{11}^{2} / 8\right)+S=0$,

where

$$
\begin{aligned}
P= & \delta^{2} \alpha^{6} L e^{2} L n^{2}, \\
Q= & \delta^{4} \alpha^{4} L e^{2} L n^{2}+\delta^{4} \alpha^{4} \varepsilon^{2}\left(L e^{2}+L n^{2}\right) \\
& -\alpha^{6} L e^{2} L n^{2} R a-\varepsilon \alpha^{6} L e^{2} R s\left(1+L n N_{C T}\right), \\
R= & \delta^{6} \alpha^{2} \varepsilon^{2}\left(L e^{2}+L n^{2}+\varepsilon^{2}\right)-\alpha^{4} \delta^{2} \varepsilon^{2}\left(L e^{2}+L n^{2}\right) R a \\
& -R n N_{A} \varepsilon \delta^{2} \alpha^{4} L n^{2}(L e+\varepsilon)+\varepsilon \delta^{2} \alpha^{4} L e^{2}\left(N_{C T} \varepsilon-1\right) R s \\
& -\varepsilon^{3} \delta^{2} \alpha^{4}\left(N_{C T} L n+1\right) R s \\
S= & \delta^{8} \varepsilon^{4}-\alpha^{2} \delta^{4} \varepsilon^{4} R a-R n N_{A} \varepsilon^{3} \delta^{4} \alpha^{4}(L e+\varepsilon)+\delta^{4} \alpha^{2} \varepsilon^{3} R s\left(N_{C T} \varepsilon-1\right) .
\end{aligned}
$$

\subsubsection{Heat, salt and nanoparticle mass transport}

The heat transport specified by the "thermal Nusselt number" is defined as where $B_{02}(t)$ is given by Eq. (58).

Similarly, the solutal concentration Nusselt number $N u_{C}(t)$ is found to be

$N u_{C}(t)=1-2 \pi D_{02}(t)+N_{C T}\left(1-2 \pi B_{02}(t)\right)$,

where $D_{02}(t)$ is given by Eq. (62).

The nanoparticle concentration Nusselt number is defined as

$N u_{\phi}(t)=1+\left[\frac{\int_{0}^{2 \pi / \alpha_{c}}\left(\frac{\partial \phi}{\partial z}+N_{A} \frac{\partial T}{\partial z}\right) d x}{\int_{0}^{2 \pi / \alpha_{c}}\left(\frac{\partial \phi_{b}}{\partial z}\right) d x}\right]_{z=0}$,

gives

$N u_{\phi}(t)=1+2 \pi\left(B_{02}(t)-C_{02}(t)\right)$,

where $C_{02}(t)$ is given by Eq. (60).

Agrawal et al. [49] discussed the Rayleigh Bénard convection in a nanofluid with local thermal equilibrium (LTE) and showed that the mode of mass transfer was diffusion only, but Eqs. (64) to (66) show that heat, salt and nano 
concentration all are transferred through diffusion as well as convection. Clearly transfer of heat, salt and mass all are governed by Soret parameter.

\section{Results and discussion}

In the limiting case of monodiffusive convection in a Maxwell nanofluid, the problem discussed converts to the one investigated in detail recently by Jaimala et al. [39]. Similarly, for regular fluid (in the absence of nanoparticles), it represents the established Soret driven double diffusive convection studied by Wang and Tang [32]. The results reported here are also used for comparison with those obtained for double diffusive convection in a nanofluid.

\subsection{Linear stability analysis}

Linear stability theory provides the Rayleigh number for stationary and oscillatory convection. For stationary convection, we recover the Rayleigh number deduced for monodiffusive convection in the nanofluid [see Eq. (38)] and Bénard convection in Horton-Rogers problem for a regular fluid [42]. Comparing the Rayleigh number for nanofluid given by Eq. (38) with the classical Rayleigh number $R a=4 \pi^{2}$, it is clear that if the density of nanoparticles is greater than the density of base fluid the Rayleigh number is reduced substantially causing an early convection, which is further diminished in the presence of salt [see Eq. (37)] but the inclusion of Soret effect delays the convection in comparison to the convection in its absence.

Tables 1 and 2 present a comparison between the three Rayleigh numbers for a single phase nanofluid, binary nanofluid, and the Soret effect in a binary nanofluid for stationary and oscillatory convections respectively for fixed values of the concerned parameters. Table 1 shows that $R a_{\text {(thermal) }} \geq R a_{\text {(Soret induced) }} \geq R a_{\text {(salt) }}$ everywhere in the flow domain. The critical Rayleigh number $R a^{c r}$ for the three convections are 23.879 for thermal convection, 11.879 for Soret convection and 11.379 for thermosolutal convection. It confirms the analytical result that the presence of salt in Maxwell nanofluid enhances the convection and establishes the fact that though Soret effect delays the convection still it occurs earlier in comparison to the convection set in a single phase nanofluid. Readings of Table 1 are reflected through Fig. 2a. Table 2 shows that oscillatory thermal convection for single phase fluid, binary fluid and binary fluid with Soret effect diminishes approximately after $a=3.7396,6.7636$ and 7.066 respectively. Corresponding Fig. $2 \mathrm{~b}$ predicts that the oscillatory convection sets up earliest in the single phase nanofluid and latest in binary fluid with Soret effect.
Table 1 Stationary convection: $R a$ for thermal convection, thermosolutal convection and Soret induced convection

\begin{tabular}{lcll}
\hline Wave number & $\begin{array}{l}R a \text { (thermal } \\
\text { convection) }\end{array}$ & $\begin{array}{l}R a \text { (thermo- } \\
\text { solutal convec- } \\
\text { tion) }\end{array}$ & $\begin{array}{l}R a \text { (Soret } \\
\text { induced con- } \\
\text { vection) }\end{array}$ \\
\hline 0.5 & 396.62557 & 384.126 & 384.6255729 \\
1 & 105.1483 & 92.6483 & 93.14829984 \\
1.5 & 52.282138 & 39.7821 & 40.28213815 \\
2 & 35.091482 & 22.5915 & 23.09148156 \\
2.5 & 28.574663 & 16.0747 & 16.57466337 \\
3 & 26.562441 & 14.0624 & 14.56244114 \\
3.5 & 26.940971 & 14.441 & 14.94097134 \\
4 & 28.827277 & 16.3273 & 16.82727699 \\
4.5 & 31.799534 & 19.2995 & 19.79953429 \\
5 & 35.635572 & 23.1356 & 23.63557244 \\
5.5 & 40.209344 & 27.7093 & 28.20934404 \\
6 & 45.445017 & 32.945 & 33.44501689 \\
6.5 & 51.294749 & 38.7947 & 39.29474942 \\
7 & 57.727149 & 45.2271 & 45.72714944 \\
7.5 & 64.720926 & 52.2209 & 52.72092598 \\
8 & 72.261226 & 59.7612 & 60.26122585 \\
8.5 & 80.337432 & 67.8374 & 68.33743152 \\
9 & 88.94179 & 76.4418 & 76.94179017 \\
9.5 & 98.068534 & 85.5685 & 86.06853391 \\
10 & 107.7133 & 95.2133 & 95.71329971 \\
\hline & & &
\end{tabular}

Behavior of Soret parameter with respect to Rayleigh number and wave number for stationary convection for $R s=5, R n=1$, Le $=8, \varepsilon=0.5, N_{A}=1$ in Fig. 3. From figure we can show that on increasing the Soret parameter $N_{C T}$ the critical Rayleigh number is increased and hence the Soret parameter is responsible for promoting the stability of the flow. The behavior of remaining parameters is the same as we have discussed in Jaimala et al. [48].

Figure 4a-i show the variations of Rayleigh number for linear oscillatory modes at the marginal state in $(R a, a)$ plane for the parameters $R s=0.5, R n=0.4, \varepsilon=0.4, N_{A}=1, L e=20, L n=5, \sigma=1$, $\lambda=0.1, N_{C T}=0.1$, by varying one of the parameters and keeping others fixed. It is seen that in Soret induced convection, initially $R a$ decreases very sharply and after an initial fall it increases in a wave number range $(2.42<a<4.94)$, then again it decreases to diminish. It is also observed that on increasing any of the parameters $R n, L n, N_{A}, \sigma$ and $N_{C T}$ the peaks of oscillatory curves get higher (see Fig. 4b, e-g, i) and get further down when any of $R s, L e, \varepsilon$ or $\lambda$ increases (see Fig. $4 \mathrm{a}, \mathrm{c}, \mathrm{d}, \mathrm{h}$ ). The results are in good agreement with double diffusive convection [39].

A comparison of stationary convection and oscillatory convection is shown in Fig. 5a-f for particular values of the parameters. It indicates that in all the cases 
Table 2 Oscillatory convection: Ra for thermal convection, thermosolutal convection and Soret induced convection

\begin{tabular}{llll}
\hline Wave number & $\begin{array}{l}R a \text { (thermal con- } \\
\text { vection) }\end{array}$ & $\begin{array}{l}R a \text { (thermo- } \\
\text { solutal convec- } \\
\text { tion) }\end{array}$ & $\begin{array}{l}R a \text { (Soret- } \\
\text { induced } \\
\text { convection) }\end{array}$ \\
\hline
\end{tabular}

\begin{tabular}{lc}
\hline 0.01 & $981,625.0564$ \\
0.1108 & 8028.898987 \\
0.2116 & 2225.193752 \\
0.3124 & 1038.04241
\end{tabular}

0.3124

0.4132

0.514

0.6148

0.7156

0.8164

0.9172

1.018

1.1188

1.2196

1.3204

1.4212

1.522

1.6228

1.7236

1.8244

1.9252

2.026

2.1268

2.2276

2.3284

2.4292

2.53

2.6308

2.7316

2.8324

2.9332

3.034

3.1348

3.2356

3.3364

3.4372

3.538

3.6388

3.7396

3.8404

3.9412

4.042

4.1428

4.2436

4.3444

4.4452

4.546

4.6468
1038.04241

606.2790448

401.7625971

288.5653739

219.0121957

172.9061895

140.5251877

116.7391759

98.64737213

84.51655289

73.26088575

64.16961416

56.75741818

50.67936174

45.68136594

41.57074891

38.19825793

35.44673908

33.22368304

31.45608034

30.08669487

29.0712357

28.37609984

27.97644323

27.85435228

27.99684752

28.39335939

29.03217608

29.89520061

30.9502634

32.14047053

33.37114147

34.4975558

35.32134059

35.60797648

786.081022

386.6043956

939.7515997

455.2913448

278.2386358

214.7948911

172.8446513

143.5314134

122.2958472

106.4982916

94.50819626

85.27230477

78.08653844

72.46717012

68.07449399

64.66586365

62.06589391

60.14706381

58.00889336

57.67731424

57.79239642

58.33796218

59.30953395

60.7132157

62.56510227

64.89109811

67.727052

71.11911858

75.12424006

79.8105955

85.25777859

91.55632037

98.80594496

107.1116046

116.5758611

127.2855741

139.290237

152.5690039

166.9842415

182.2226721

197.7326275

212.6785456

225.9485566

236.2567427

63.09197386

63.77036357

64.88051948

66.43026487

68.43683187

70.92671284

73.93577489

77.50955633

81.70364737

92.22707267

98.71913101

106.154776

114.6333409

124.2523647

135.096435

147.2193604

160.6173749

175.191537

190.6995294

206.7018764

222.5160562

237.2029621
58.81682455
Table 2 (continued)

\begin{tabular}{|c|c|c|c|}
\hline Wave number & $\begin{array}{l}R a \text { (thermal con- } \\
\text { vection) }\end{array}$ & $\begin{array}{l}R a \text { (thermo- } \\
\text { solutal convec- } \\
\text { tion) }\end{array}$ & $\begin{array}{l}\mathrm{Ra} \text { (Soret- } \\
\text { induced } \\
\text { convection) }\end{array}$ \\
\hline 4.7476 & & 242.3603645 & 249.6179599 \\
\hline 4.8484 & & 243.3544156 & 258.5521393 \\
\hline 4.9492 & & 238.9360957 & 262.9575522 \\
\hline 5.05 & & 229.5106325 & 262.1982537 \\
\hline 5.1508 & & 216.0783482 & 256.2288924 \\
\hline 5.2516 & & 199.9605152 & 245.6158153 \\
\hline 5.3524 & & 182.4948123 & 231.3880838 \\
\hline 5.4532 & & 164.8128259 & 214.7884962 \\
\hline 5.554 & & 147.739789 & 197.0278867 \\
\hline 5.6548 & & 131.7945849 & 179.1170921 \\
\hline 5.7556 & & 117.2447399 & 161.7961379 \\
\hline 5.8564 & & 104.1775189 & 145.5393574 \\
\hline 5.9572 & & 92.56461688 & 130.6018067 \\
\hline 6.058 & & 82.31188585 & 117.0780891 \\
\hline 6.1588 & & 73.29361993 & 104.9565872 \\
\hline 6.2596 & & 65.37426826 & 94.16220383 \\
\hline 6.3604 & & 58.42110289 & 84.58680565 \\
\hline 6.4612 & & 52.31088621 & 76.10928453 \\
\hline 6.562 & & 46.93280042 & 68.60786491 \\
\hline 6.6628 & & 42.18918261 & 61.9670581 \\
\hline 6.7636 & & 37.99505851 & 56.08113315 \\
\hline 6.8644 & & & 45.25288317 \\
\hline 6.9652 & & & 36.27066926 \\
\hline 7.066 & & & 28.81416711 \\
\hline 7.1668 & & & \\
\hline
\end{tabular}

86.58401665 the oscillatory convection occurs only for a particular range of wave number and there exist no wave number where the critical Rayleigh number exists. It occurs at the wave number beyond which the oscillatory convection ceases to exist.

\subsection{Nonlinear stability analysis}

\subsubsection{Steady analysis}

To detect the onset of convection in steady state, the effect of increasing Rayleigh number on heat, concentration and mass transport across the layer is important. It is noticed that the steady state flow depends upon the Soret parameters but is independent of $\sigma$ and $\lambda$.

Figures 7 and 8 portray the effect of Ra on both the Nusselt numbers $N u_{C}$ and $N u_{\phi}$ respectively for the values of the parameters $R s, L e, R n, \varepsilon, L n, N_{A}$ and $N_{C T}$ by varying one of the parameters.

It is observed from Fig. 6 that, as Rayleigh number increases, thermal Nusselt number increases very sharply i.e. heat transfer increases at a sharp rate and as Rayleigh 
(a)

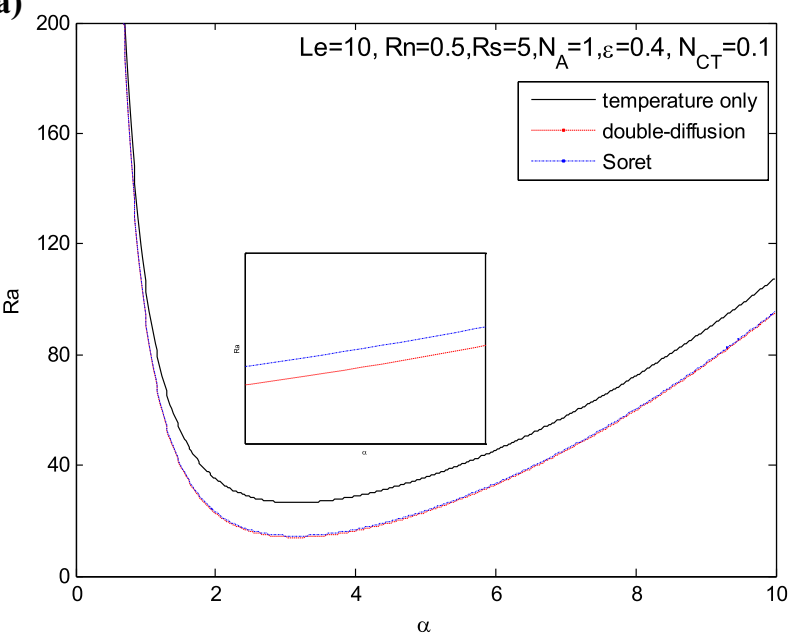

(b)

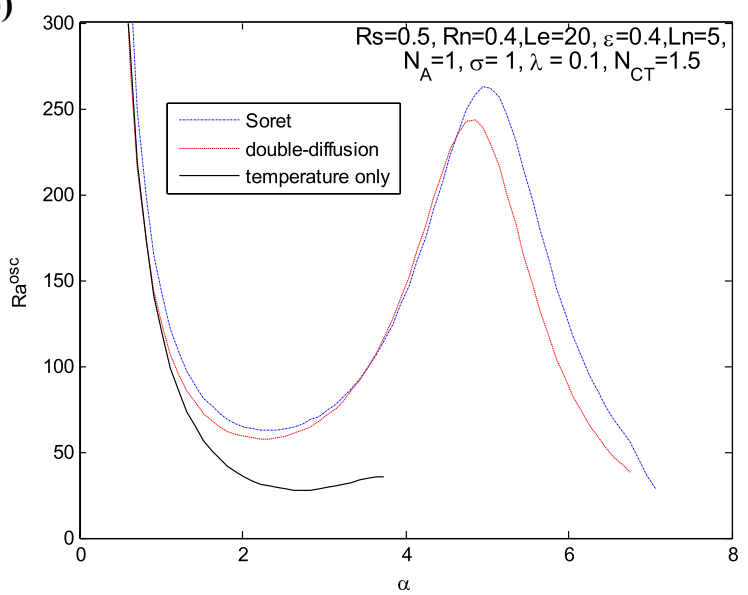

Fig. 2 a Stationary convection for thermal, solutal and Soret induced convection. b Oscillatory convection for thermal, solutal and Soret induced convection

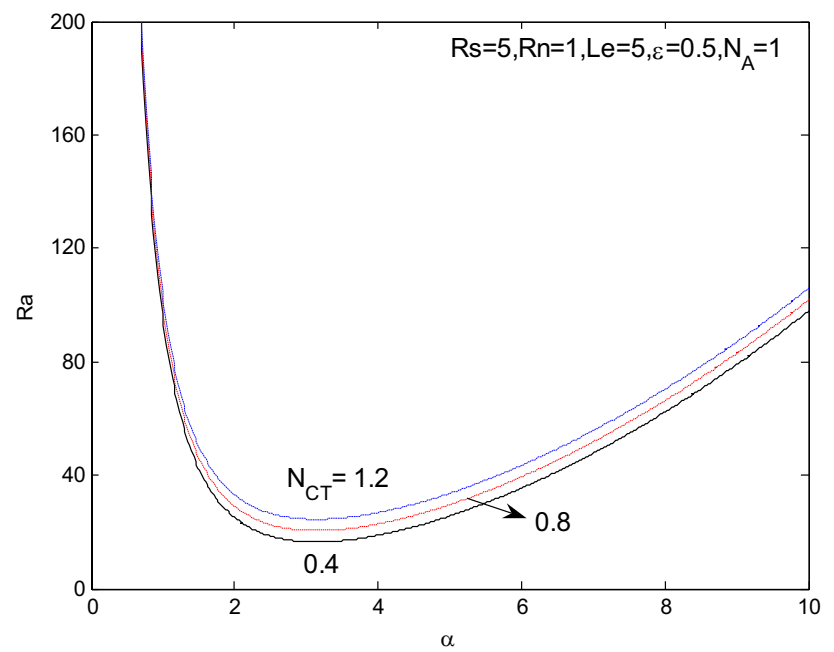

Fig. $3 R a$ versus a for linear stationary convection for $N_{C T}$ number is increased further, it attains a steady state. On increasing any of the values of the parameters $R s, R n, \varepsilon$, and $N_{A}$ the heat transfer is increasing before attaining a steady state [48]. Also the parameters $L e$ and $L n$ separately have reverse effect on heat transfer. It is decreased on increasing any of the parameters though it again becomes constant for large values of Rayleigh number [48]. It is interesting to note that Fig. 6 portrays a dual character of Soret parameter $N_{C T}$. Initially, on increasing $N_{C T}$, heat transfer is decreased then starts to increase for a fixed range of Rayleigh number ( $<R a=33.5$ and at that point, value of $\mathrm{Nu}=1.15$ ) and finally reaches at a constant value for $\mathrm{Ra}>300$ (approximately). Figure 7 shows that, on increasing $R a$, initially the salt is transferred in large amount but it becomes constant for large values of Rayleigh number ( $>250$ approximately). The parameters $R s$, $R n, L n, N_{A}$ and $N_{C T}$ individually are responsible to increase the salt transfer. Figure 7c interprets that the Lewis number Le predicts very surprising behavior as for $L e \geq 5.9$ salt transfer is decreased by a substantial amount. Further Fig. $7 \mathrm{~d}$ depicts the behavior of porosity parameter $\varepsilon$. It shows that on increasing the medium porosity, the salt transfer is decreased. It is to be noted that on increasing the porosity, heat transfer is increased but the amount of salt transfer is decreased. The behavior of $\varepsilon$ remains unaltered even in the presence of thermophoresis. It is observed from Fig. 7e, $g$ that salt transfer is enhanced by a considerable amount for $L n \geq 5$ and $N_{C T} \geq 0.5$.

Figure $8 \mathrm{a}-\mathrm{g}$ indicate that initially mass transfer is increased with the Rayleigh number but a sharp fall is noticed with increasing $R a$ which finally reaches to a steady state. In double diffusive convection, concentration Nusselt number is depending upon the parameters as $R s, R n, L e, \varepsilon, L n, N_{A}$ and $N_{C T}$. It is seen that the parameters $R s, R n, \varepsilon$ and $N_{A}$ have dual character (Fig. $8 \mathrm{a}, \mathrm{b}, \mathrm{d}$, f). It is to be noticed that the parameters $R n, \varepsilon$ and $N_{A}$ had the same nature in monodifusive convection in a nanofluid. On increasing $R s, R n$ and $N_{A}$ individually, the mass transfer is increased and after a certain Rayleigh number it starts to decrease. An increase in porosity initially results in a fall in mass transfer which is boosted for higher values of Rayleigh number. Surprisingly, Le changes its behavior and shows a dual nature. It is clear from Fig. $8 \mathrm{c}$ that initially mass transfer is increased by increasing Le but after a certain value of $R a$ it inhibits the transfer of mass. In thermal convection, it was noticed to increase the mass transfer. It is observed from Fig. $8 \mathrm{e}, \mathrm{g}$ that thermosolutal Lewis number $L n$, and Soret parameter $N_{C T}$ follow the nature of porosity.

A comparative study of the three Nusselt numbers $\mathrm{Nu}$, $\mathrm{Nu} u_{C}$ and $N u_{\Phi}$ is shown in Fig. 9. It is observed that nanoparticles are transferred most actively. Though in the initial range of $R a(<80)$ salt is transferred more rapidly 
(a)

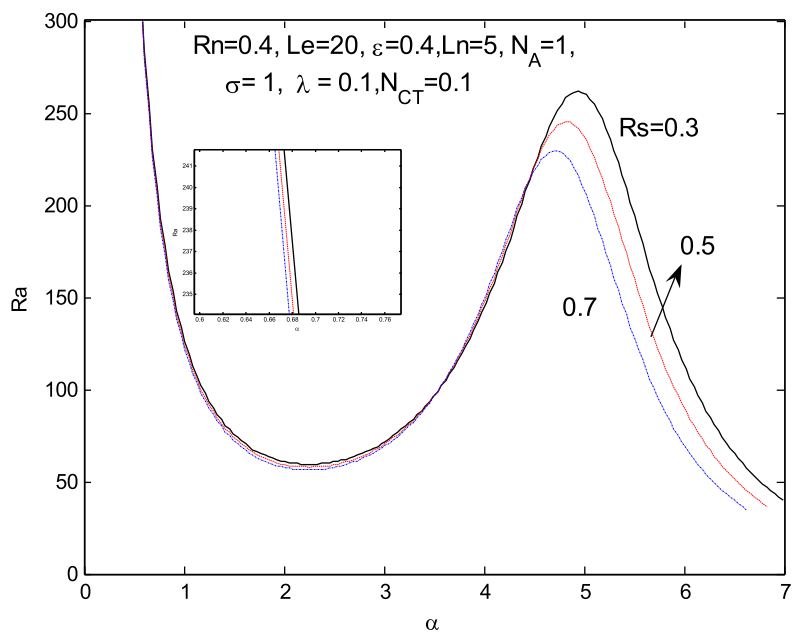

(c)

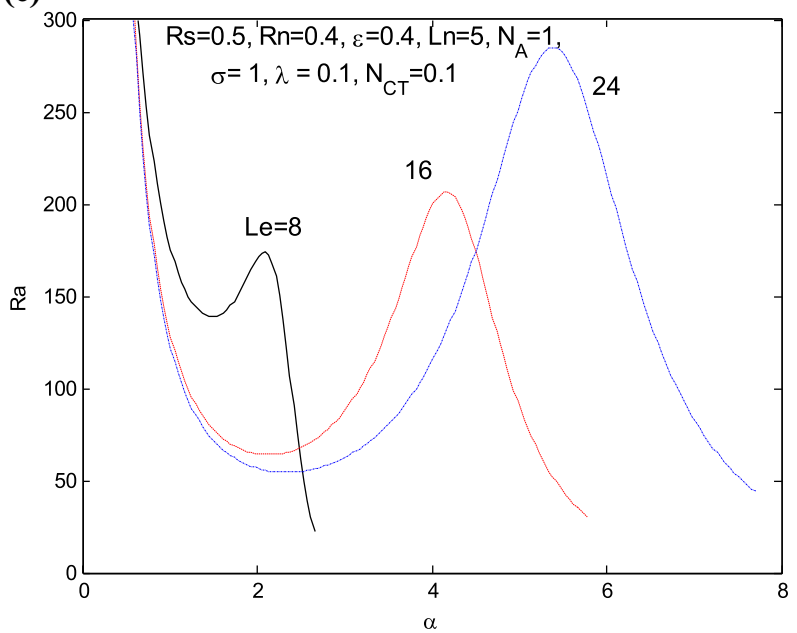

(e)

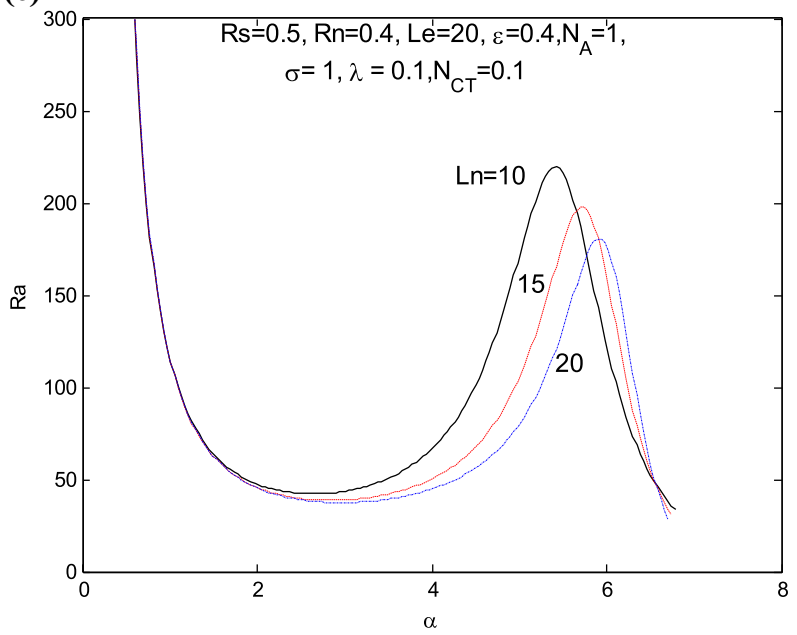

(b)

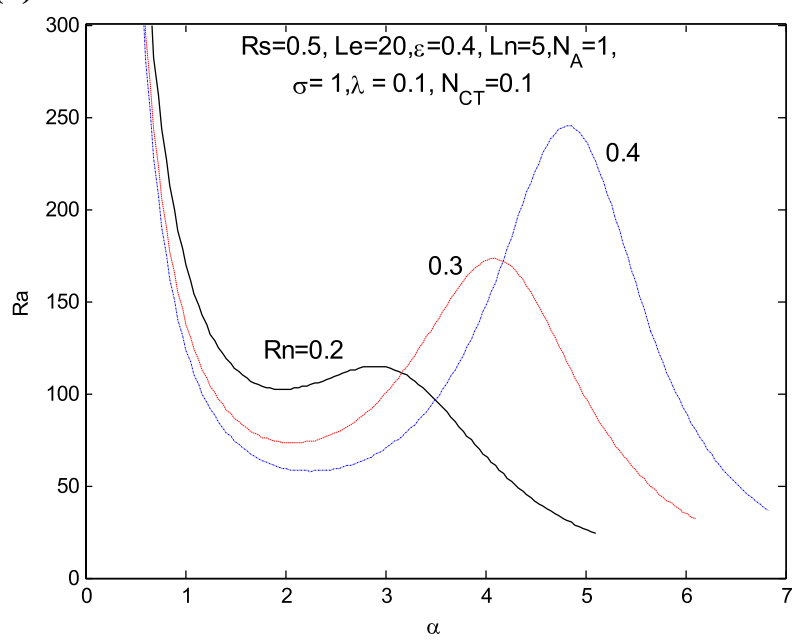

(d)

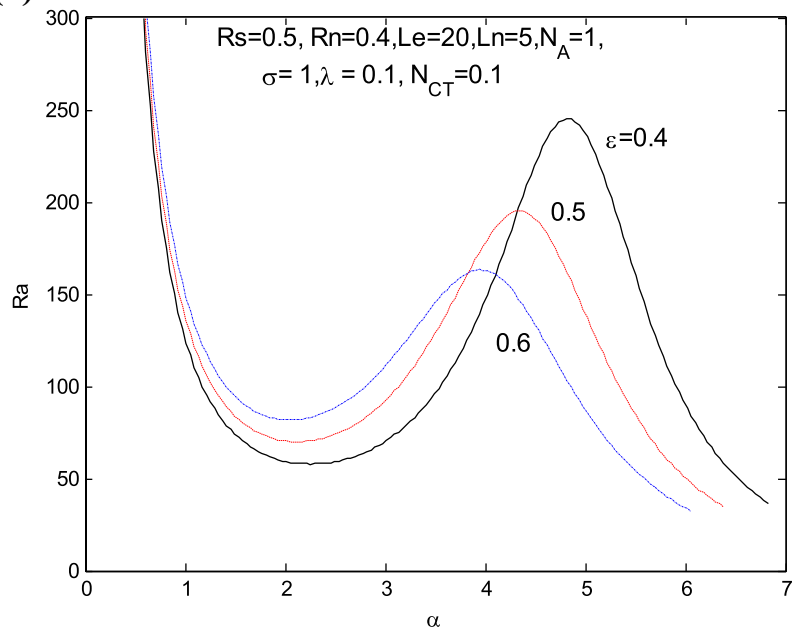

(f)

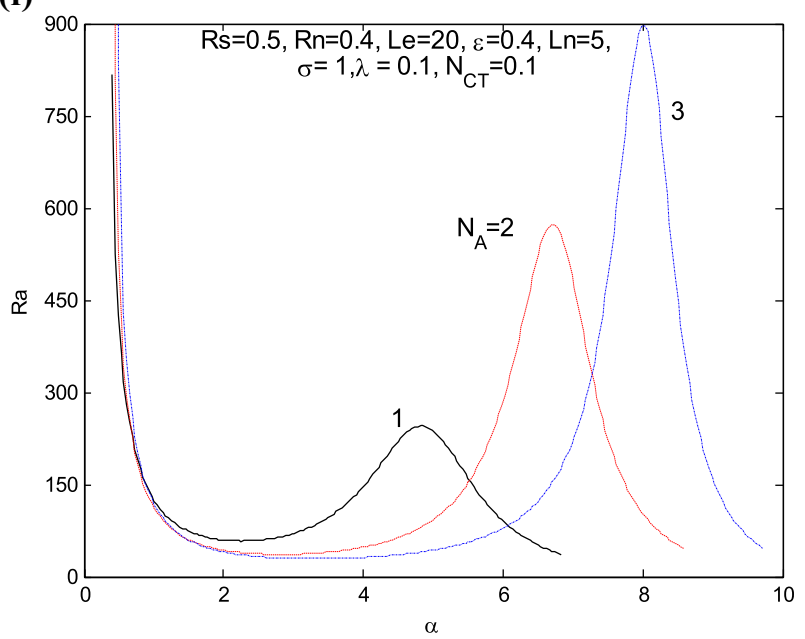

Fig. 4 Ra versus a for linear oscillatory curve for a Rs b Rn c Le $\mathbf{d} \varepsilon \mathbf{e} \operatorname{Ln} \mathbf{f} N_{A} \mathbf{g} \sigma \mathbf{h} \lambda \mathbf{i} N_{C T}$ 
(g)

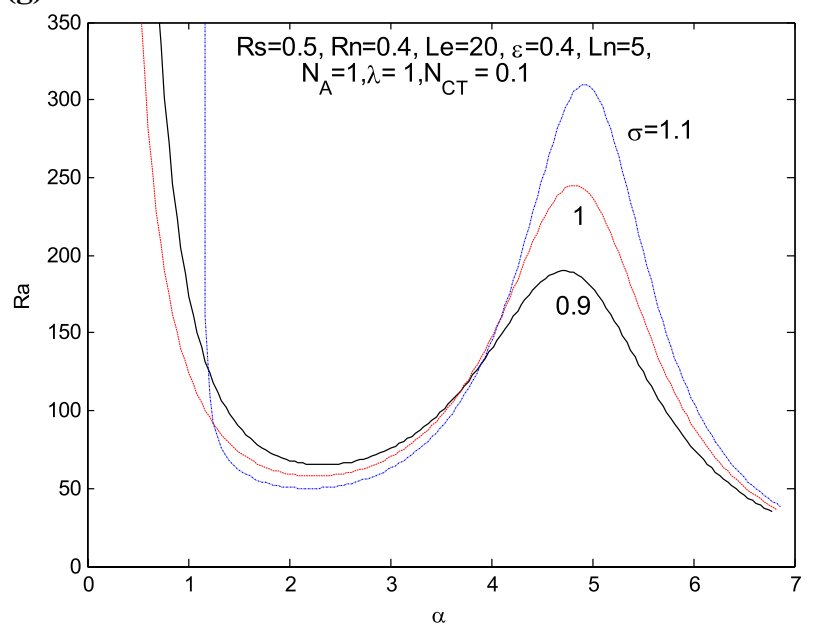

(i)

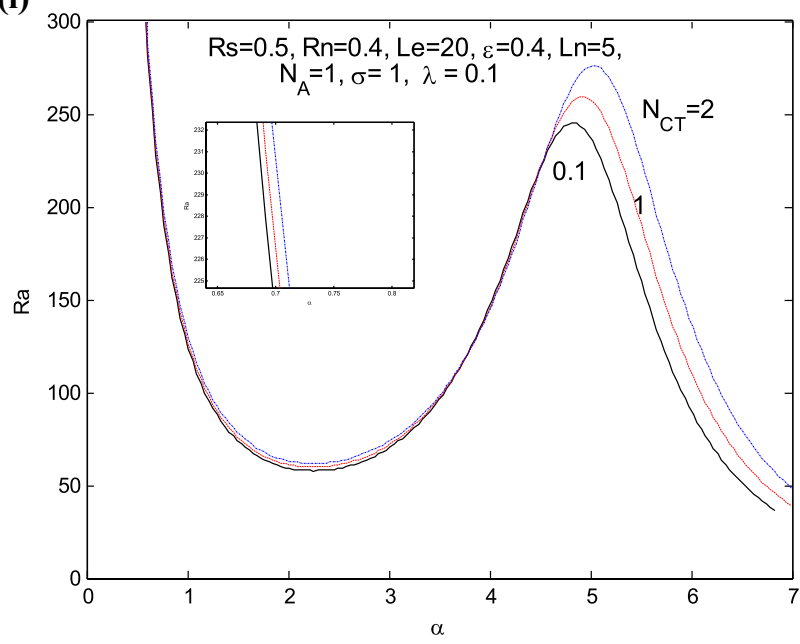

(h)

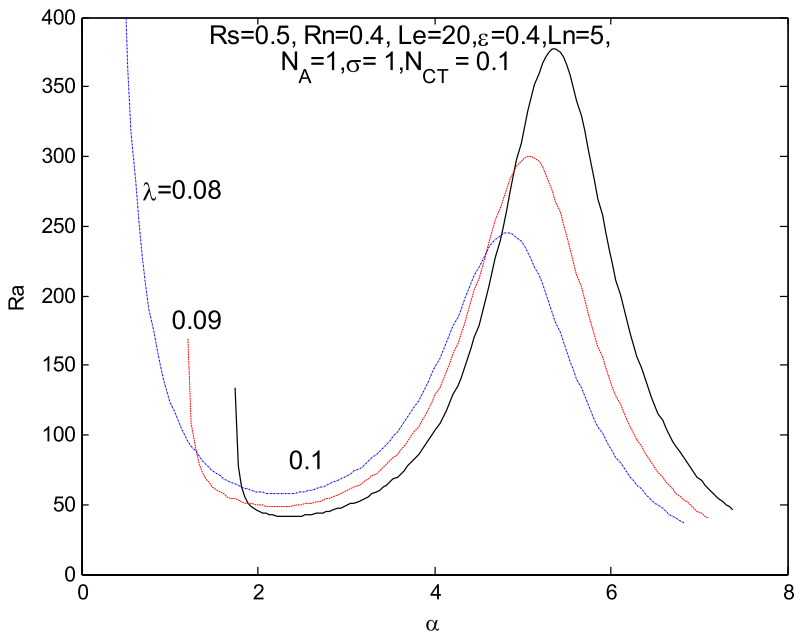

Fig. 4 (continued)

than heat but for Ra $>80$ it faces a fall and is decreased by a large amount in comparison to the transfer of heat.

In order to observe the intensity of heat, salt and mass transfer according to their existence in the three convections; thermal convection, double diffusive convection and Soret induced convection, the behavior of $\mathrm{Nu}, \mathrm{Nu} u_{C}$ and $\mathrm{Nu}$ verses $R a$ is shown separately in Fig. 10. Figure 10a, portraying Nu shows that heat is transferred most actively in Soret induced convection. It is also observed that in spite of a delay in heat transfer in terms of Rayleigh number in monodiffusive convection, it reaches to a steady state analogous to the double diffusive convection. Figure 10b, showing $\mathrm{Nu}_{C}$ indicates that Soret effect decreases the transfer of salt and Fig. 10c shows that mass is transferred most actively in mono-diffusive convection and is least active in double diffusion convection but Soret effect helps it to enhance.
Figure 11 shows the time independent evolution of the flow patterns in terms of stream lines, isotherms, isonanoconcentrations and isohalines for different values of Rayleigh number for the fixed values of the parameters $R s=2$, $R n=0.5, L e=2.5, \varepsilon=0.4, L n=2, N_{A}=2, N_{C T}=0.1$. It is clear from Figs. $11 \mathrm{a}, \mathrm{b}$ that the magnitude of stream function increases with increasing $R a$. The stream lines move alternately identical in the subsequent cells but move in the directions opposite to each other. It indicates the symmetrical formation of the convective cells. Isotherms shown in Fig. 11c, d predict that as Rayleigh number increases the convective mode of transfer of heat becomes weak and it starts to transfer through conduction. The patterns of isonanoconcentration show that the transfer of mass through diffusion also falls weak with increasing $R a$ (see Fig. 11e, f). Figure $11 \mathrm{~g}$, h portray that when the Rayleigh number is increased the transfer of salt concentration through both 
(a)

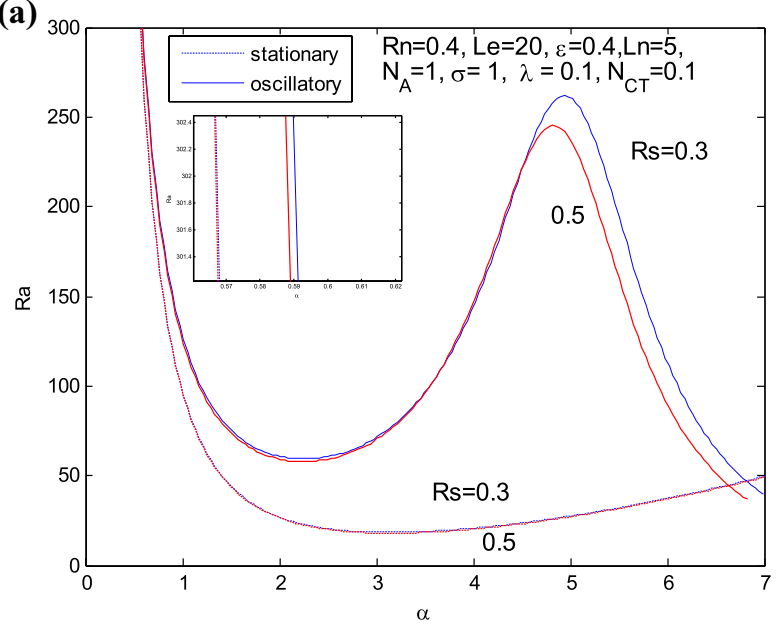

(c)

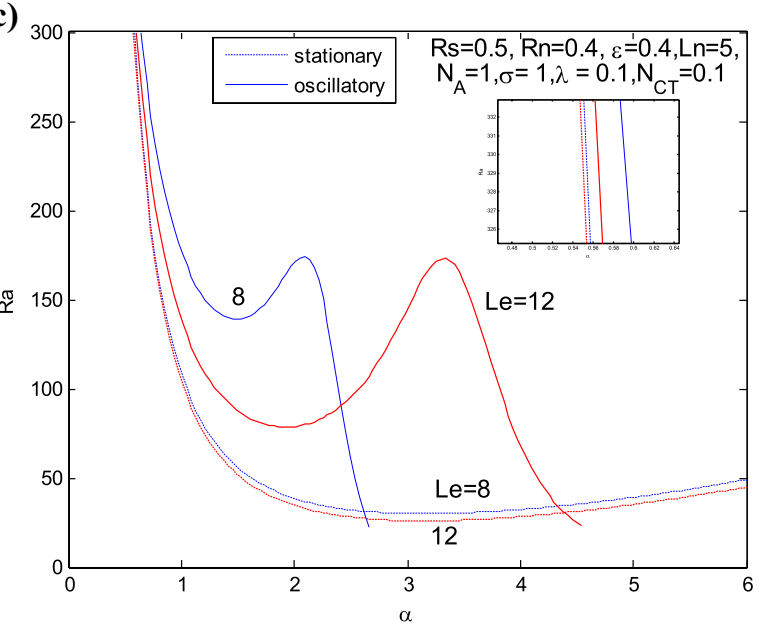

(e)

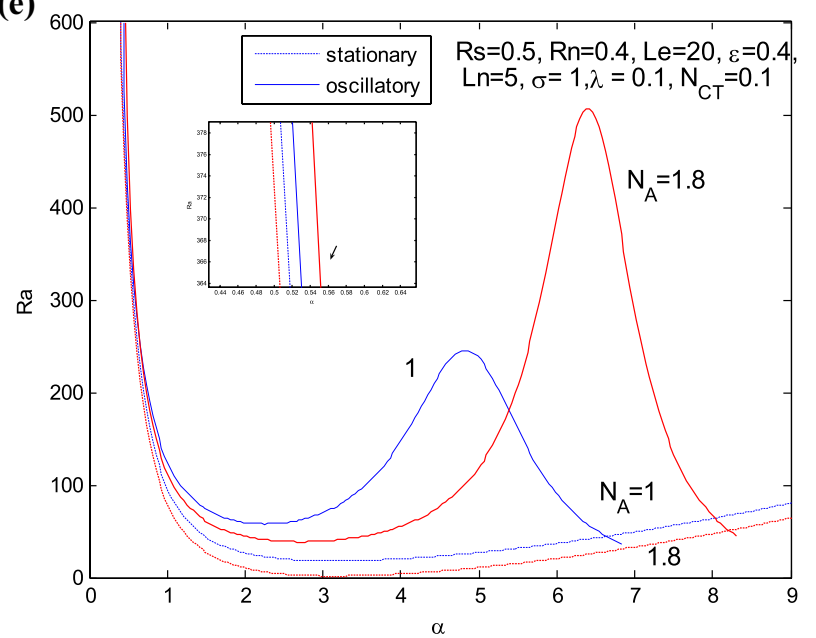

(b)

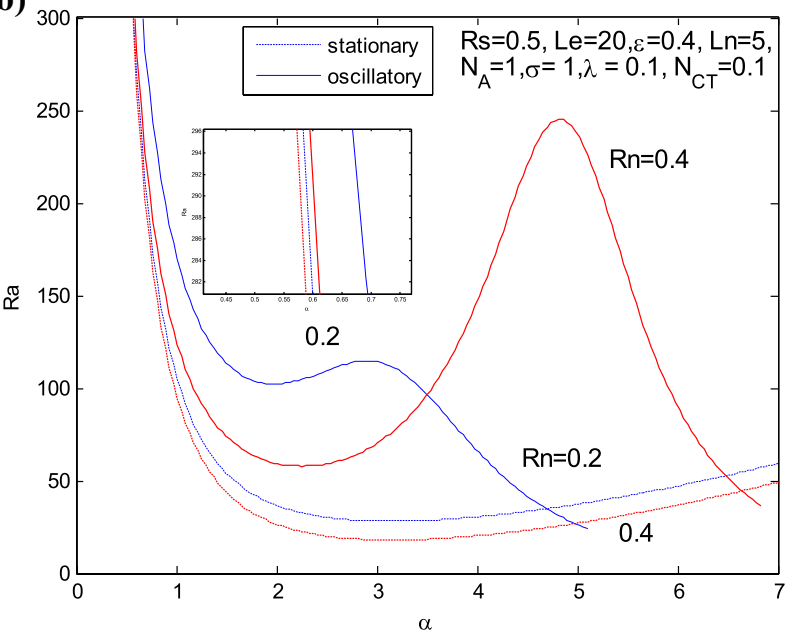

(d)

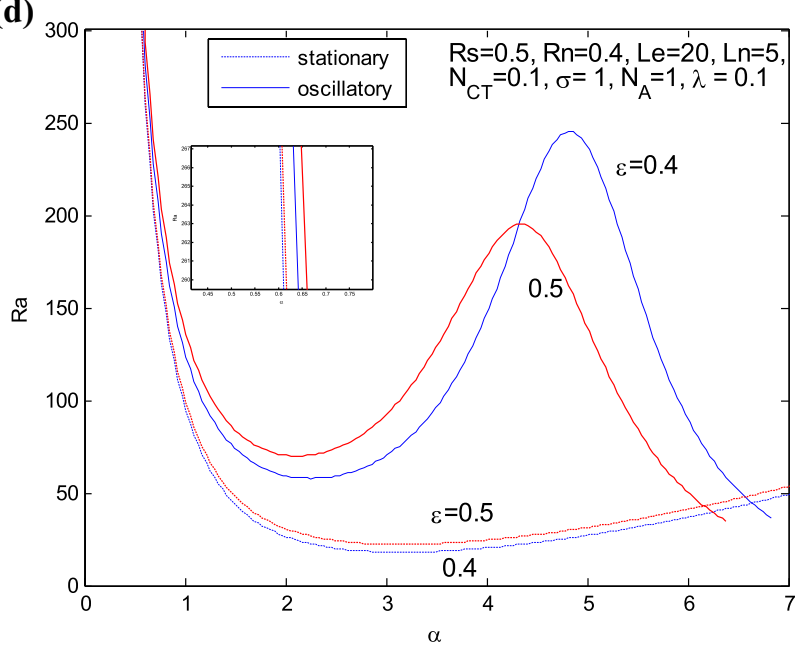

(f)

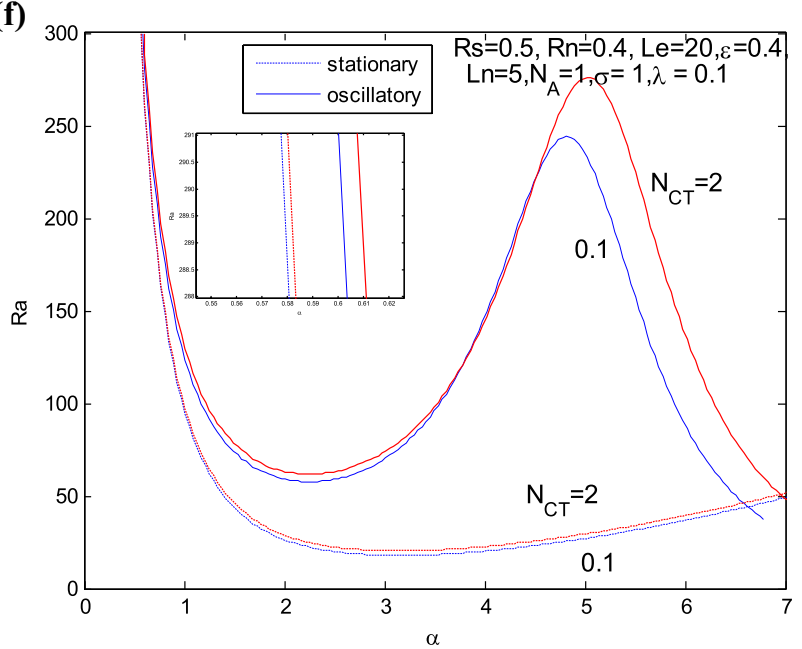

Fig. 5 Comparison of stationary and oscillatory convection for a $R s \mathbf{b} R n \mathbf{c} L e \mathbf{d} \varepsilon \mathbf{e} N_{A} \mathbf{f} N_{C T}$ 


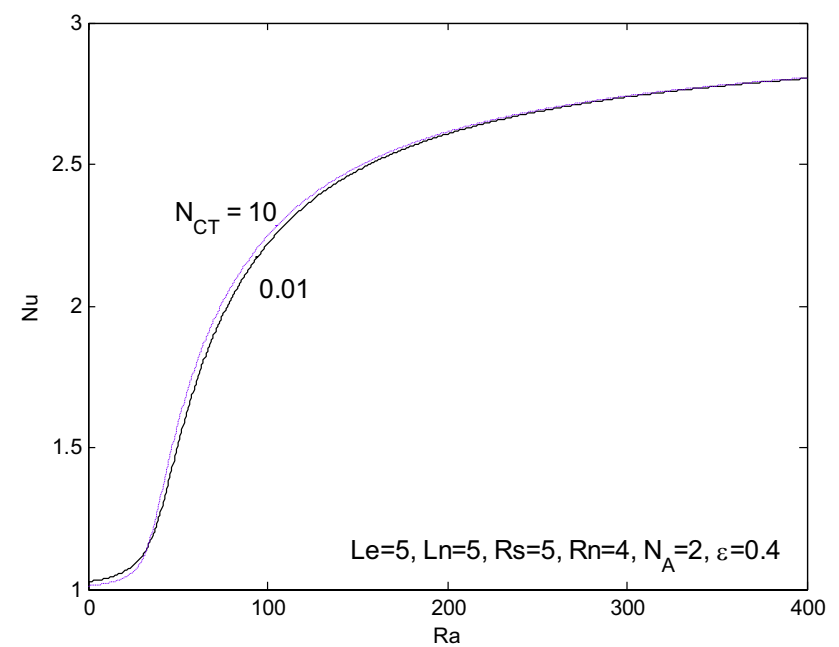

Fig. 6 Thermal Nusselt number $N u$ versus Rayleigh number $R a$ for $N_{C T}$

convection and diffusion persists but the amplitudes of the two gradually become weak.

\subsubsection{Unsteady analysis}

In the unsteady state of motion, the time history of all the three convection parameters $\mathrm{Nu}$, $N u_{C}$ and $N u_{\phi}$ are plotted in Figs. 12, 13 and 14 for the case $R s=5, R n=4, L e=10, \varepsilon=0.4, L n=10$, $N_{A}=2, \sigma=0.8, \lambda=0.5$ and $N_{C T}=0.1$, when one of the parameters is varied and the others are kept constant. It is observed that initially for small values of time, the transfer of heat, salt concentration and nanoparticles increase sharply and then after an immediate sharp decline attain a steady state through a path of rigorous oscillations. Figure $12 \mathrm{a}, \mathrm{c}, \mathrm{h}$ show the unpredictable behavior of $R s, L_{e}$ and $\lambda$. At one instant of time these parameters increase the heat transfer, while at the other these are responsible to decrease it. Figure $12 \mathrm{~d}$, e shows that $\varepsilon$ and $L n$ separately increase the amount of heat transfer as the time passes. From Fig. 12b, $f$ it is observed that transfer of heat decreases with an increase in any of the two parameters $R n$ and $N_{A}$. Further heat is transferred through oscillations only for lower values of the parameters. Figure $12 \mathrm{~g}$ show that initially higher values of $\sigma$ lowers the transfer of mass then it is increased for a small range of time and after that it is again declined through oscillations. In case of Soret parameter higher values of it increases the transfer of heat through oscillations after a decline in it for a particular range of time (Fig. 12i). Figures 13 and 14 show that for all parameters salt and mass are transferred through strong unsteady oscillatory patterns.
Figure 15 shows the time dependent stream lines, isotherms, isonanoconcentration and isohalines for $R s=2$, $R n=0.5$, Le = 5.5, $\varepsilon=0.4, L n=5, N_{A}=2, \sigma=0.5, \lambda=0.1$, $N_{C T}=0.1$. It is be noted from Fig. $15 \mathrm{a}, \mathrm{b}$ that the magnitude of stream function decreases with time. The sense of motion of stream lines in the subsequent cells is alternately identical and opposite in direction. It again indicates the symmetrical formation of the convective cells. Figure $15 c$, d predict that as the time passes the convective mode of transfer of heat becomes weak and conduction starts near the walls. Figure $15 \mathrm{e}-\mathrm{h}$ show that mass of nanoparticles and salt which were initially transferred through convection are also transferred through diffusion as the time passes.

\section{Conclusion}

Linear and non-linear analyses of Soret-driven convection in a horizontal porous layer saturated with a Darcy-Maxwell nanofluid with top heavy suspension is investigated theoretically as well as numerically. The problem is solved for destabilizing thermal and solutal gradients (i.e. when the layer is heated and soluted from below) with positive Soret coefficient. A modified Darcy-Maxwell model suggested by Khuzhayorov et al. [1] subjected to Stefan's flow condition has been used. The results are compared with the existing relevant studies. The important results are summarized as follows:

\subsection{Stability analysis (linear)}

\subsubsection{Non oscillatory or stationary convection}

- The convection at the marginal state depends upon almost all-important parameters including Soret parameter. It is unaffected by the relaxation time of Maxwell fluid.

- The convection which was set up earlier due to the presence of salt in a nanoparticle suspended fluid is delayed by the Soret parameter but still earlier convection is noticed in Soret driven phenomena in comparison to the monodiffusive convection in a nanofluid.

- Concentration Rayleigh Number Rn, solutal Rayleigh Number Rs, thermosolutal Lewis number $L n$ and modified diffusivity ratio $N_{A}$ are the parameters promoting the convection. On the other hand, the porosity parameter $\varepsilon$ and the Soret parameter $N_{C T}$ are responsible for postponing it. 
(a)

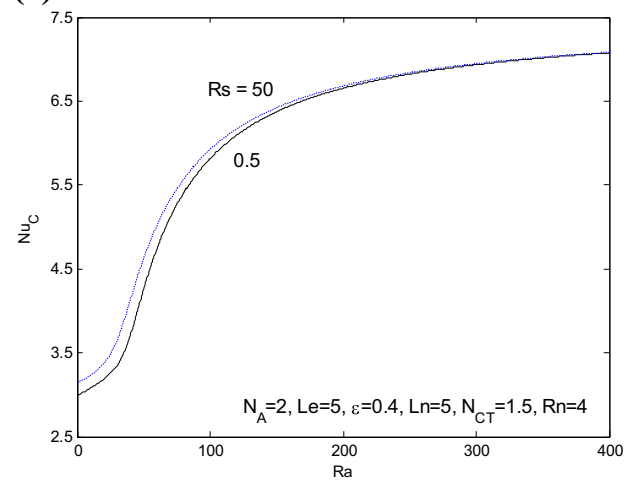

(c)

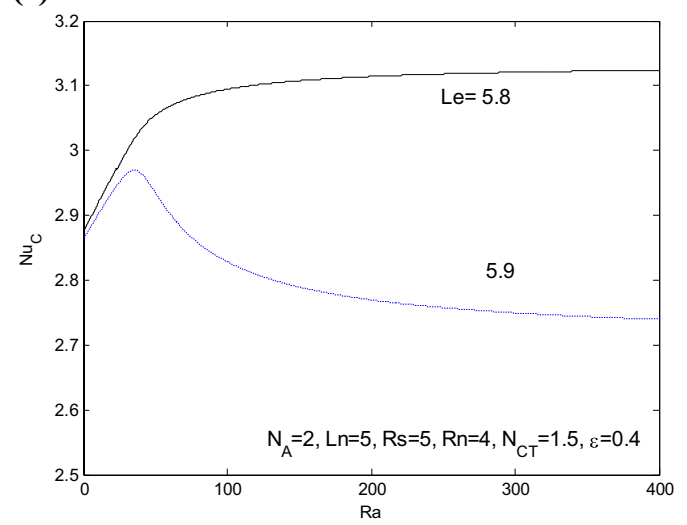

(e)

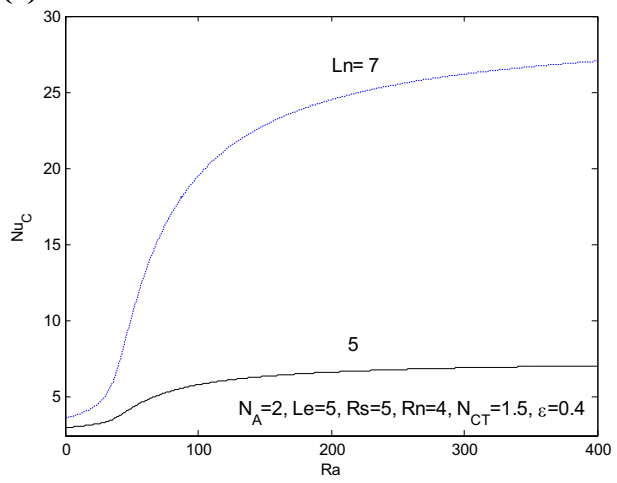

(g)

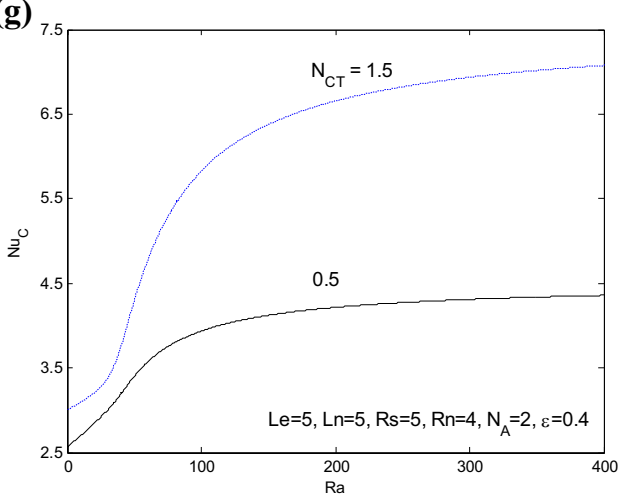

(b)

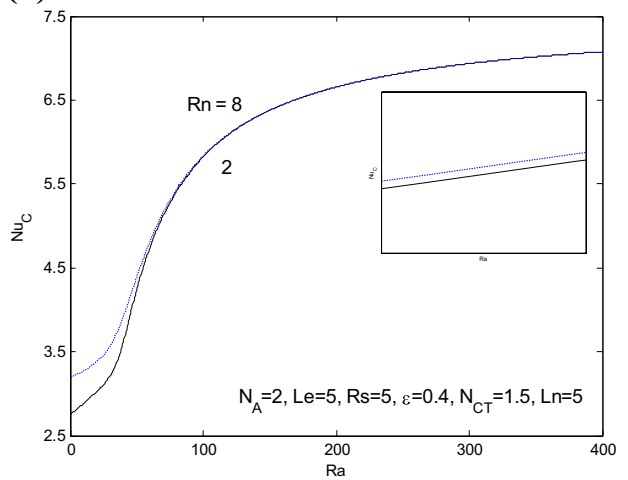

(d)

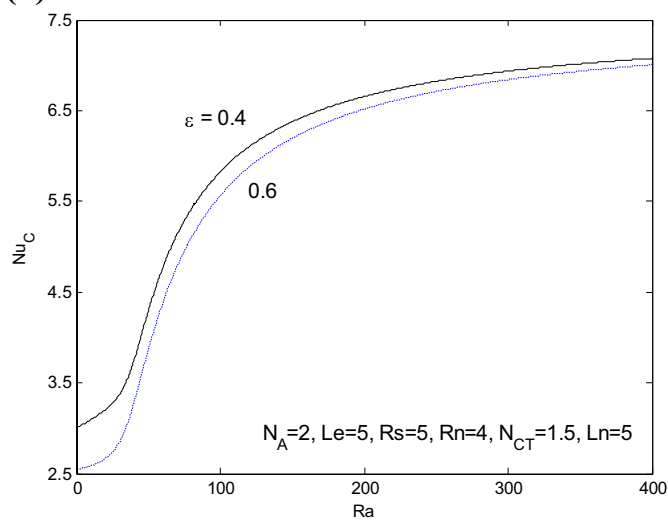

(f)

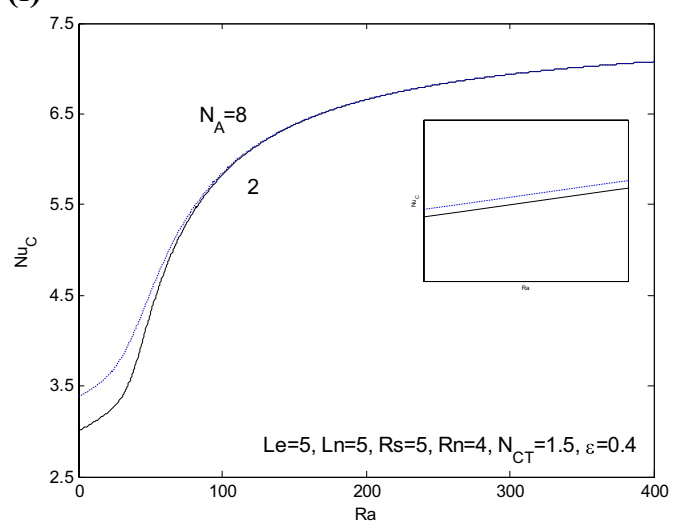

Fig. $7 N u_{C}$ versus $R a$ for a $R s$ b Rnc $L e$ d $\varepsilon$ e $\ln \mathbf{f} N_{A} \mathbf{g} N_{C T}$

\section{SN Applied Sciences}


(a)

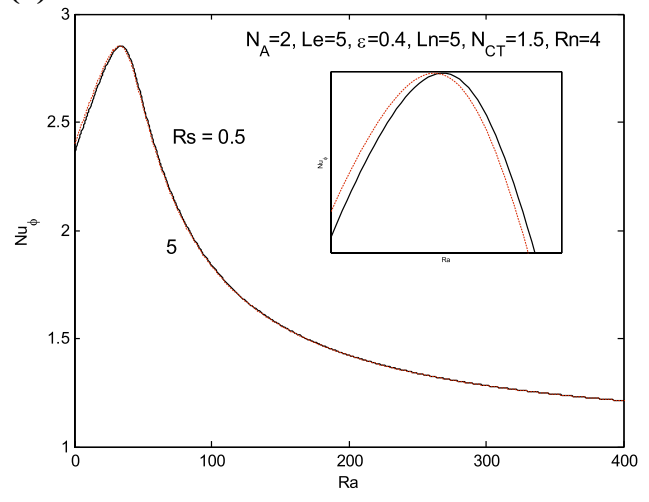

(c)

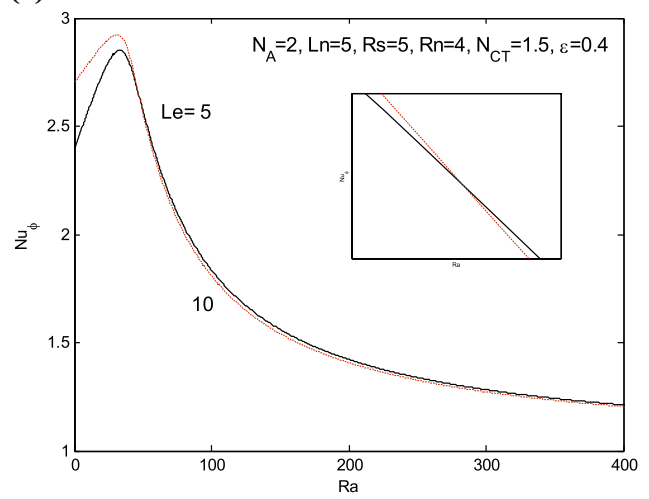

(e)

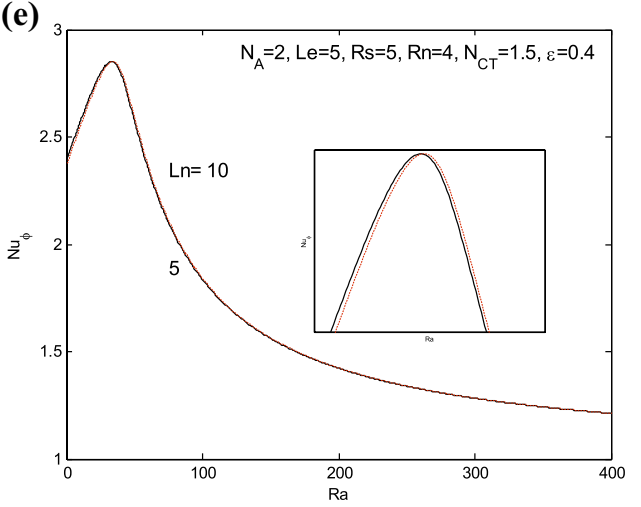

(g)

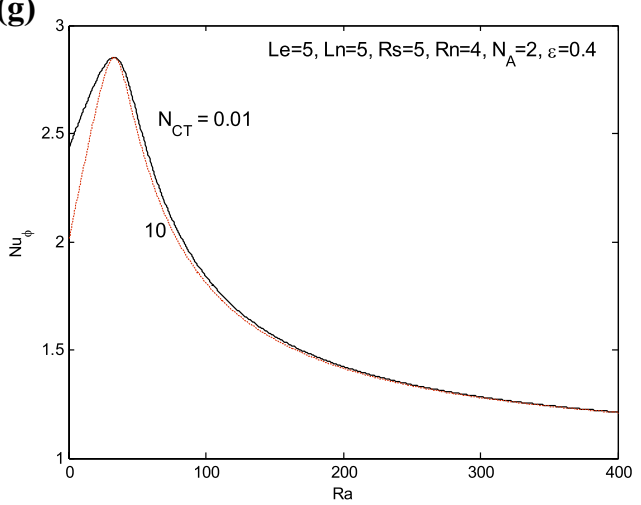

(b)

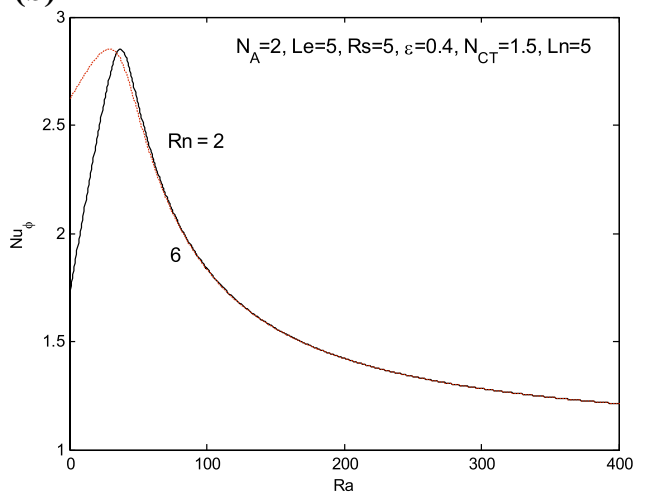

(d)

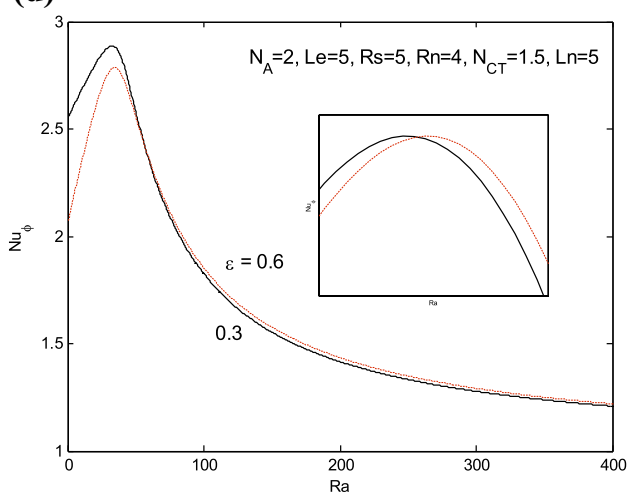

(f)

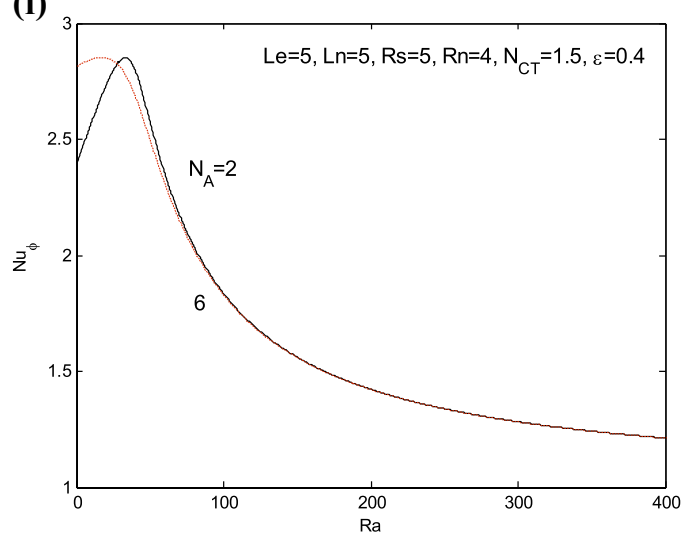

Fig. $8 N u_{\phi}$ versus $R a$ for a $R s$ b $R n$ c $L e \mathbf{d} \varepsilon$ e $L n \mathbf{f} N_{A} \mathbf{g} N_{C T}$ 


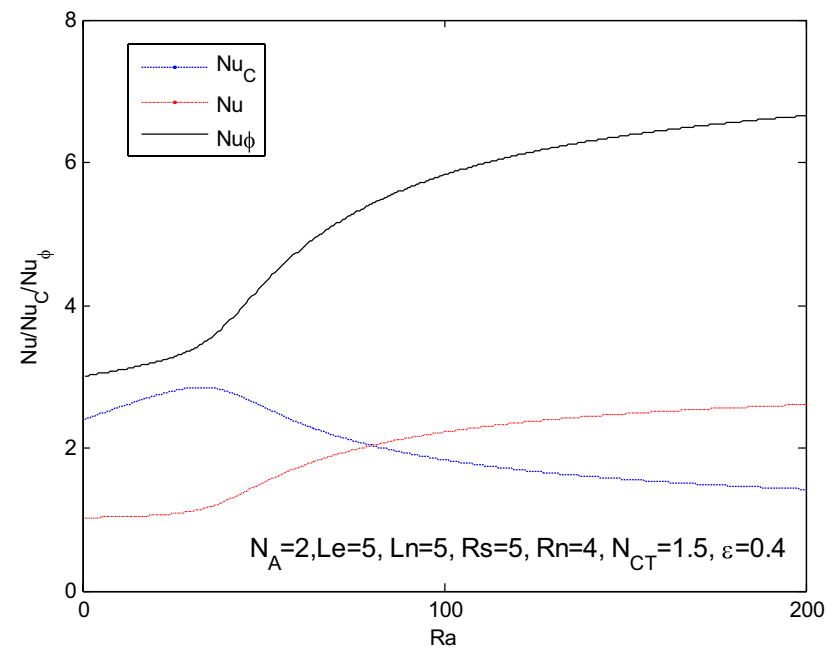

Fig. 9 Comparison between $\mathrm{Nu}, \mathrm{Nu} u_{C}$ and $\mathrm{Nu}_{\phi}$ for Soret-induced convection

(a)

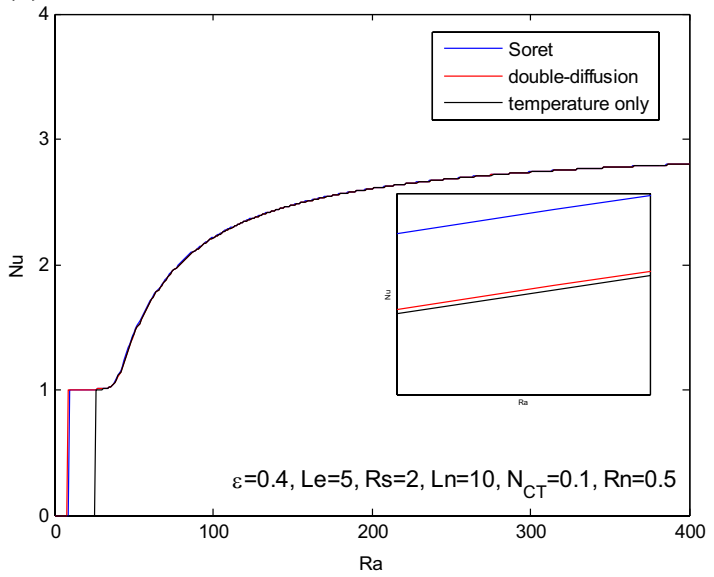

\subsubsection{Oscillatory convection}

- The convection occurs only for a specific wave number range.

- Critical Rayleigh number exists for the wave number where oscillatory convection ceases and shifts to the stationary convection which occurs earlier to it.

- In the common wave number range of the three convections; thermal convection, thermosolutal convection and Soret-driven convection, the convection in a single-phase fluid which was last to set in stationary convection sets up earliest in oscillatory convection. It sets up latest in binary fluid with Soret effect. Thus, the thermal oscillatory convection is delayed by the presence of salt which is further postponed by the presence of thermophoresis.

(c)

(b)

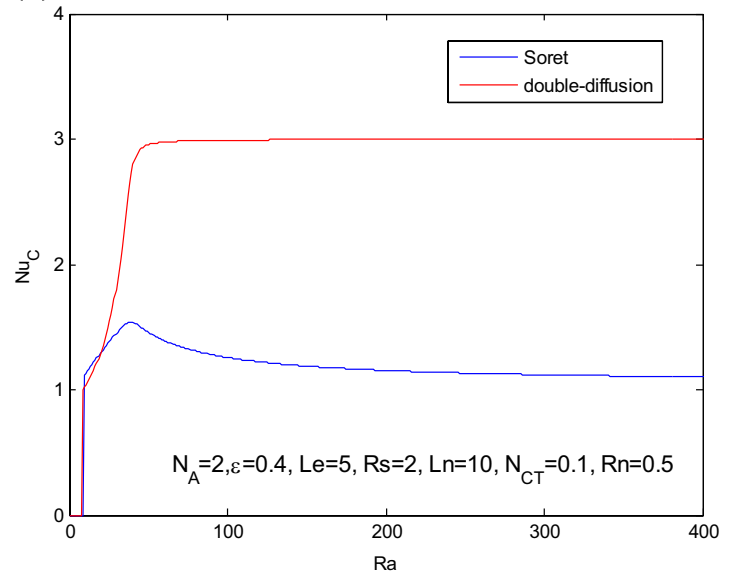

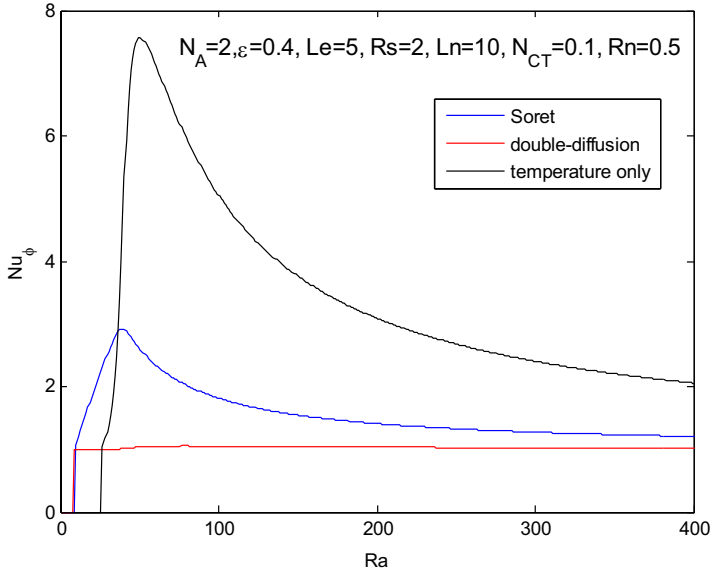

Fig. 10 a $(\mathrm{Nu}, \mathrm{Ra})$ curves for monodiffusive, double diffusive and Soret induced convection. $\mathbf{b}(\mathrm{Nu}, \mathrm{Ra})$ curves for double diffusive and Soret induced convection. c $\left(N u_{\phi^{\prime}}, R a\right)$ curves for monodiffusive, double diffusive and Soret induced convection

\section{SN Applied Sciences}




$$
R a=4 \pi^{2}
$$
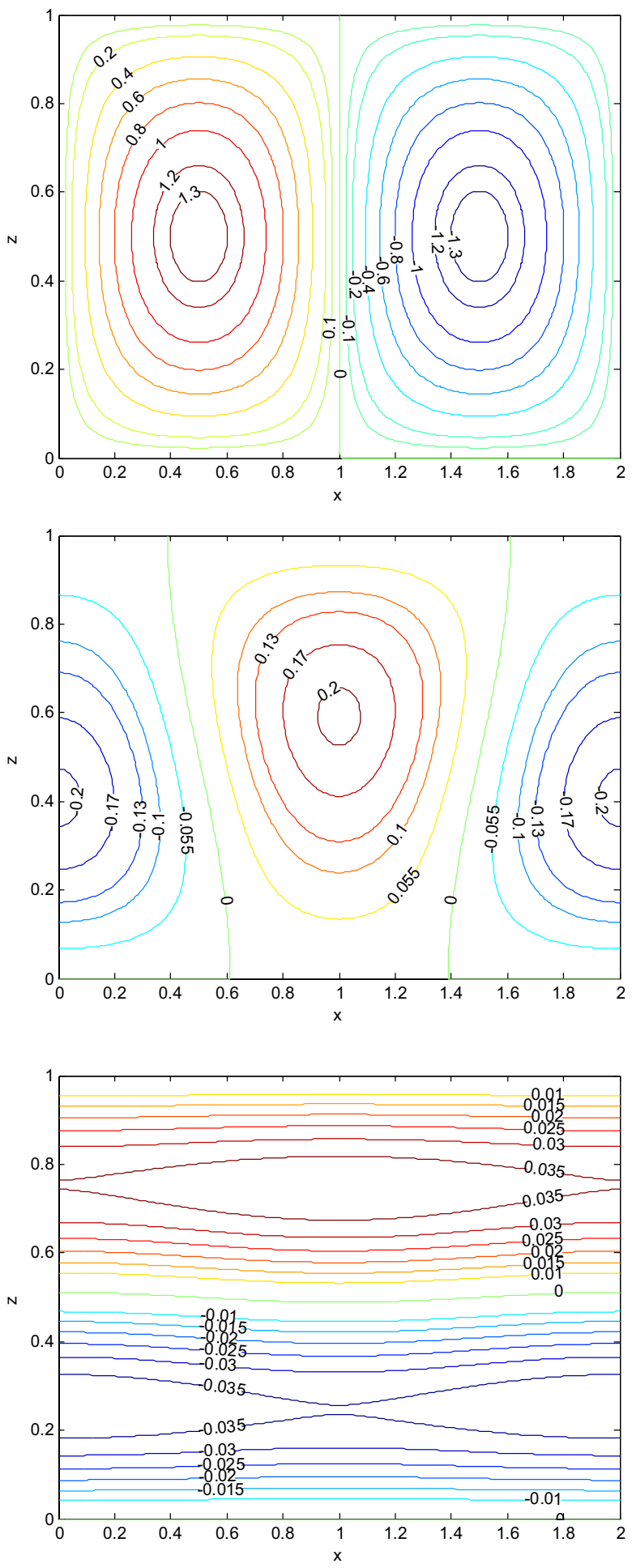

$R a=40 \pi^{2}$
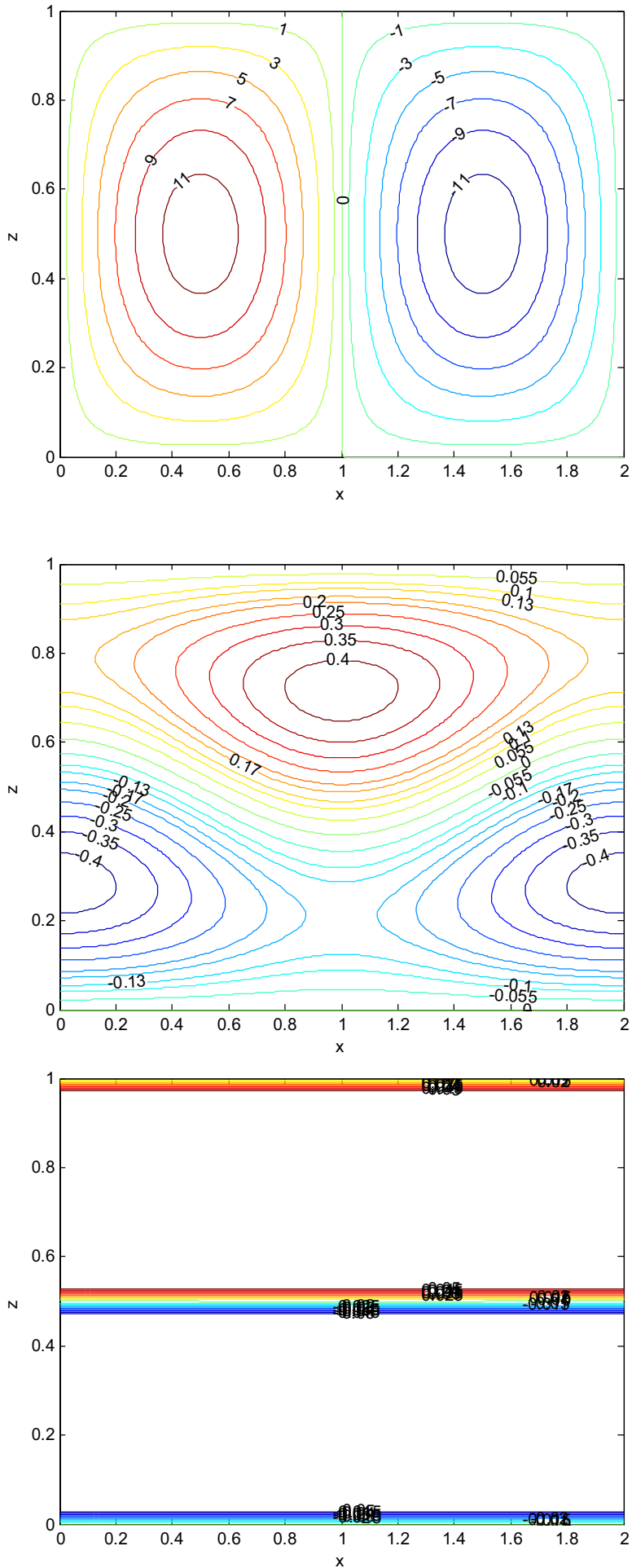

Fig. 11 Streamlines, isotherms, isonanoconcentration, isohalines for $R s=2, R n=0.5, L e=2.5, \varepsilon=0.4, L n=2, N_{A}=2, N_{C T}=0.1$ 


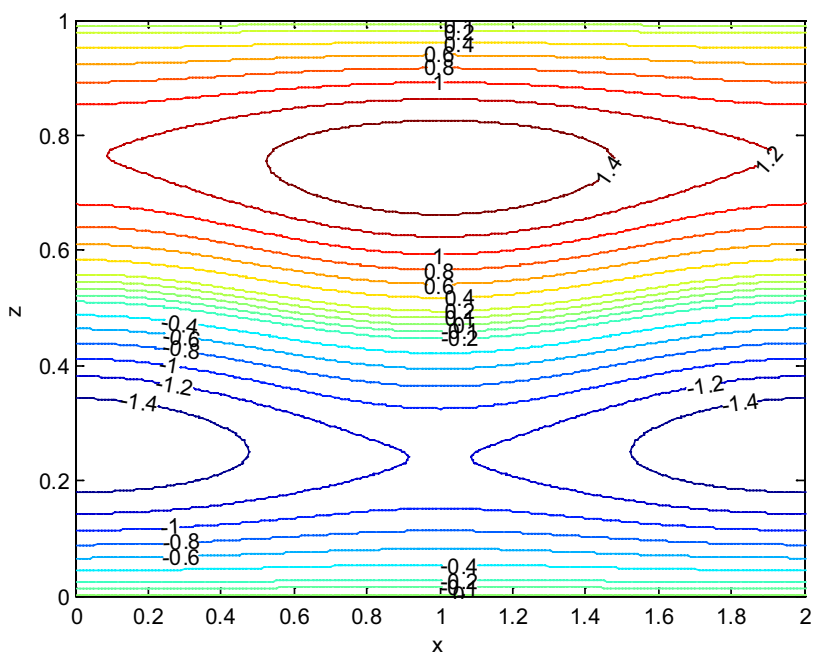

Fig. 11 (continued)

\subsection{Stability analysis (nonlinear)}

\subsubsection{Steady state}

- The convection is independent of $\sigma$ (heat capacity ratio of the medium and the fluid) and the relaxation parameter $\lambda$.

- The three Nusselt numbers: thermal Nusselt number $\mathrm{Nu}$, solutal Nusselt number $\mathrm{Nu}$ and nanoparticle concentration Nusselt number $\mathrm{Nu}_{\phi}$ depend upon the Soret parameter.

- Heat and salt transfers are enhanced continuously with increasing $R a$ while after initial increment in mass transfer; a downfall is noticed in it. Finally, the heat, mass and salt transfers attain a steady state.

- $R_{S}, R n, \varepsilon$ and $N_{A}$ are the parameters promoting the heat transfer while $L e$ and $L n$ have the reverse effect. The Soret parameter $N_{C T}$ has a dual character.

- $R_{S}, R n, L n, N_{A}$ and $N_{C T}$ are responsible for active salt transport but a substantial enhancement is noticed for $L n>5$ and $N_{C T}>0.5$. Salt transfer is decreased due to $\varepsilon$ and $L e$ but again it has a sudden fall at large values of Le (>5.9).

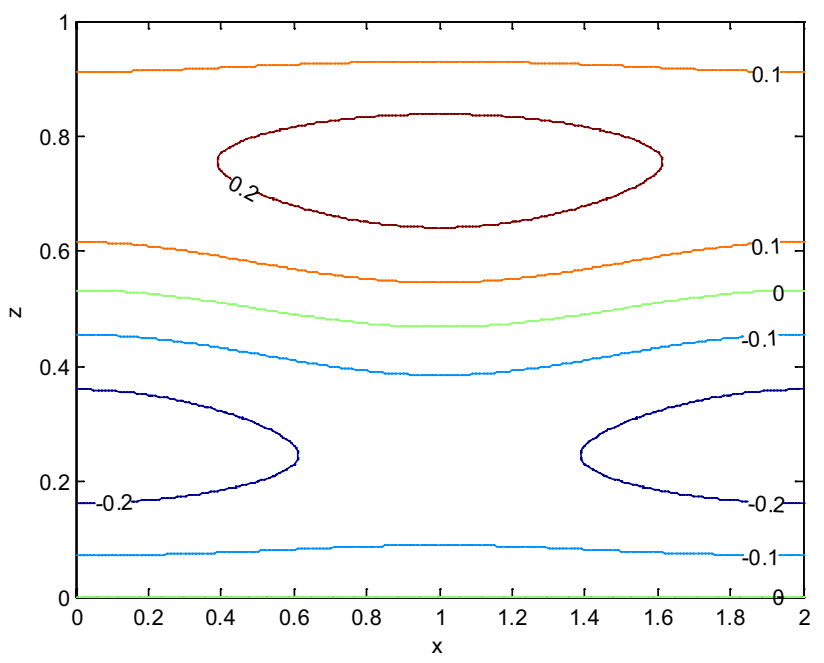

- In Soret governed convection, among heat, salt and mass, transport of nanoparticles is most active.

- Among monodiffusive, double diffusive and Soret governed convection, heat transfer is most active in Soret induced convection, salt transfer dominates in double diffusive convection and mass transfers most actively in mono-diffusive convection.

- Heat, mass and salt are transferred through diffusion and convection as well.

\subsubsection{Unsteady analysis}

- The parameters $\varepsilon$ and $L n$ individually, increase the amount of heat transfer while $R n, \sigma$ and $N_{A}$ are responsible to decrease it as the time passes.

- The salt and the mass transfer are through vigorous oscillatory patterns.

- The magnitude of stream function decreases with time and heat, mass and salt all are transferred through diffusion and convection both. 
(a)

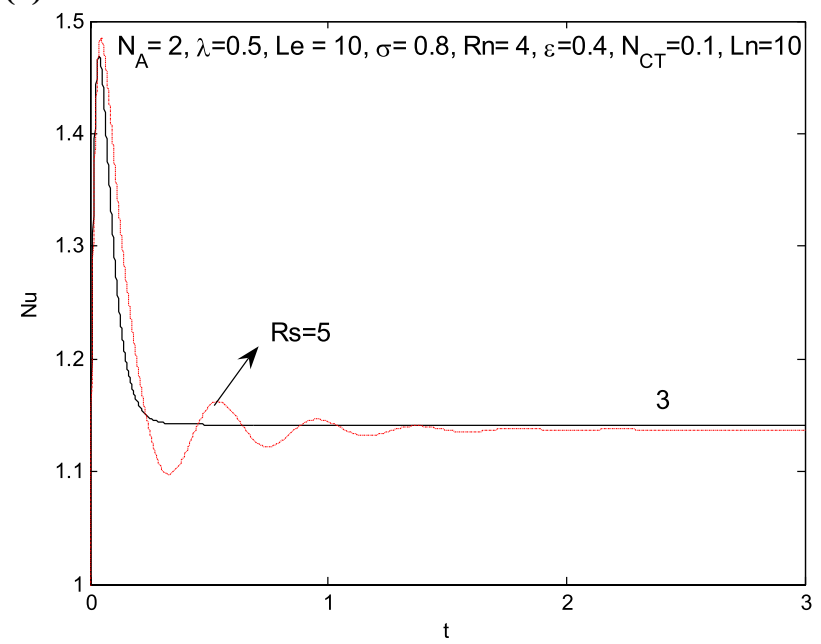

(c)

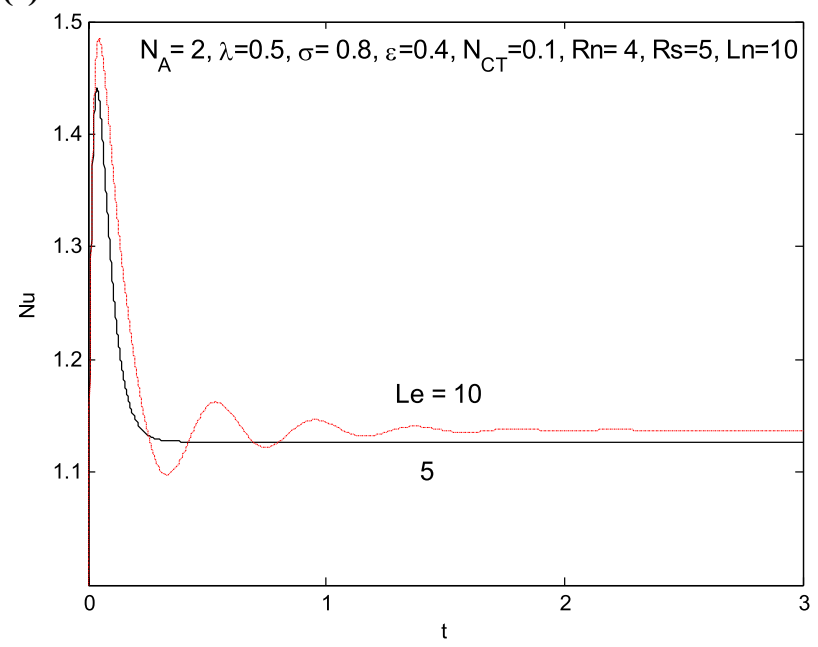

(e)

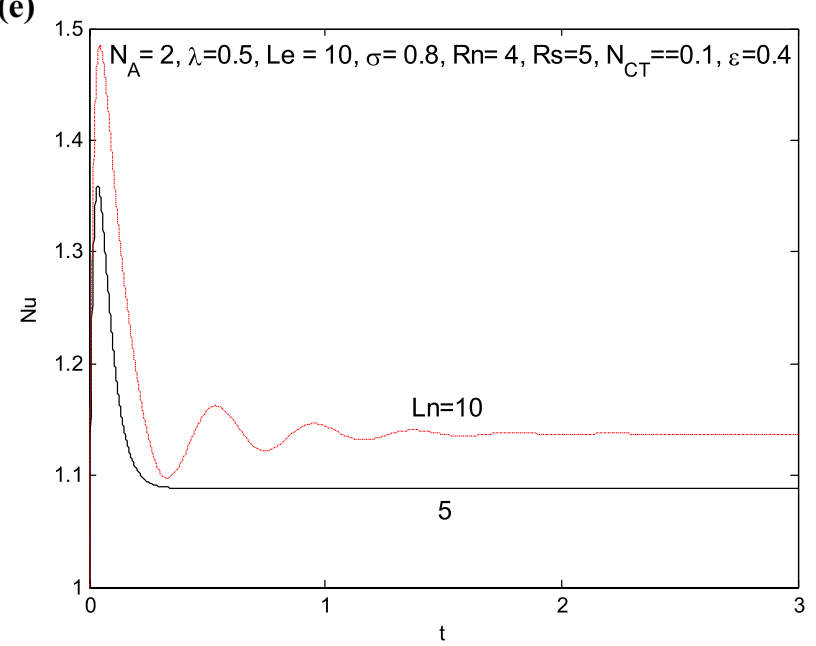

(b)

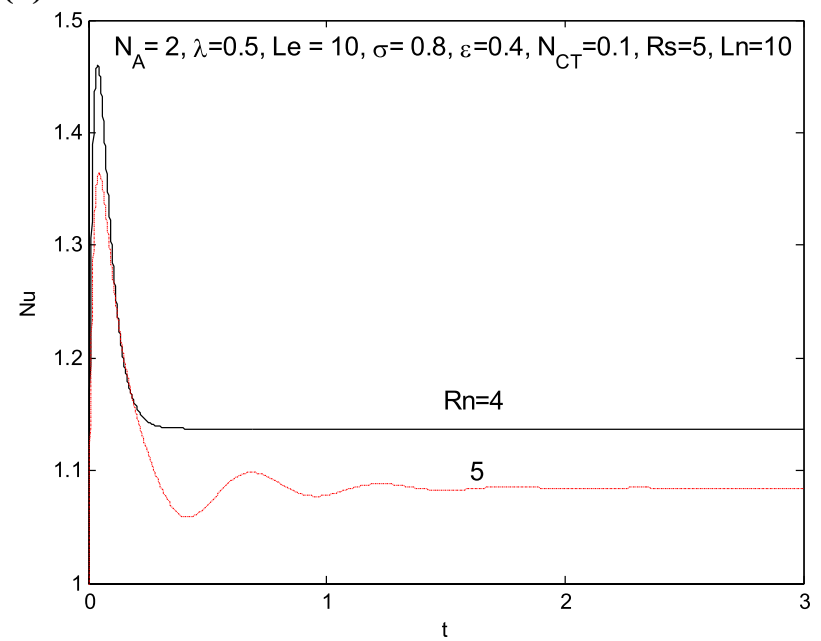

(d)

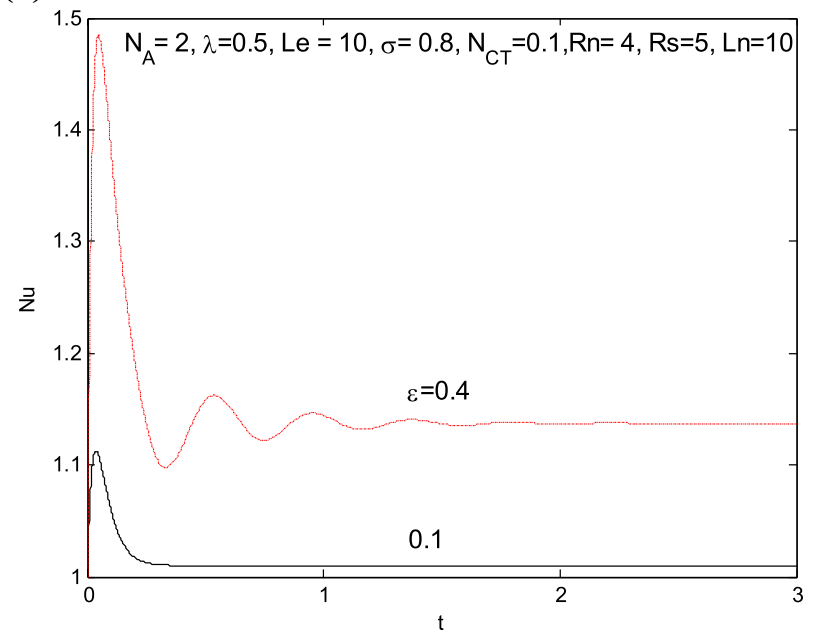

(f)

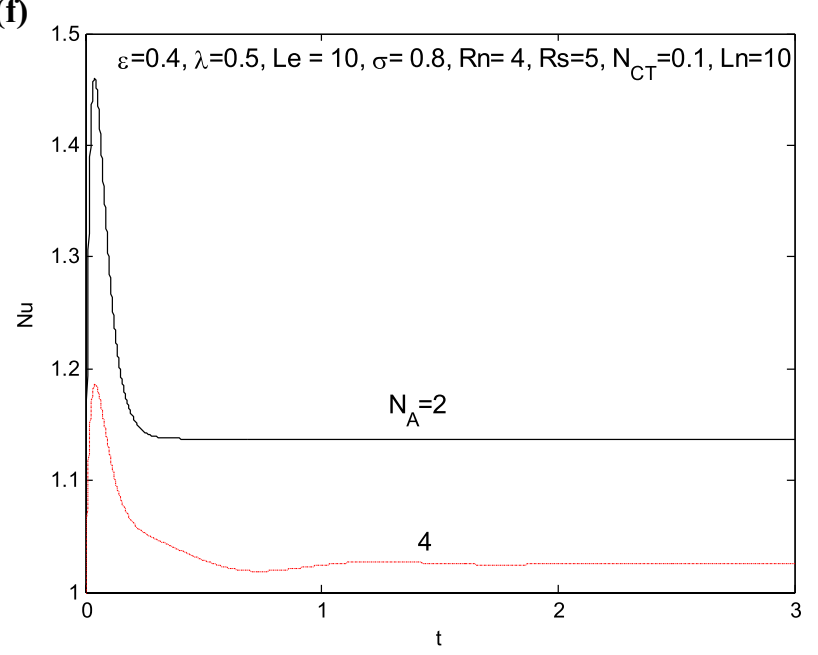

Fig. $12 N u$ versus $t$ for a $R s$ b $R n \mathbf{c} L e \mathbf{d} \varepsilon$ e $L n \mathbf{f} N_{A} \mathbf{g} \sigma \mathbf{h} \lambda \mathbf{i} N_{C T}$ 
(g)

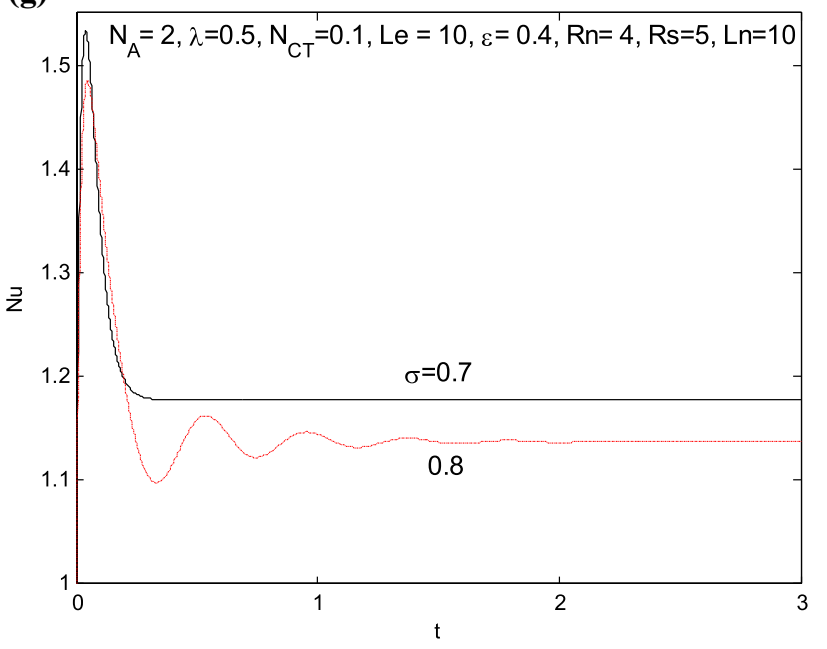

(i)

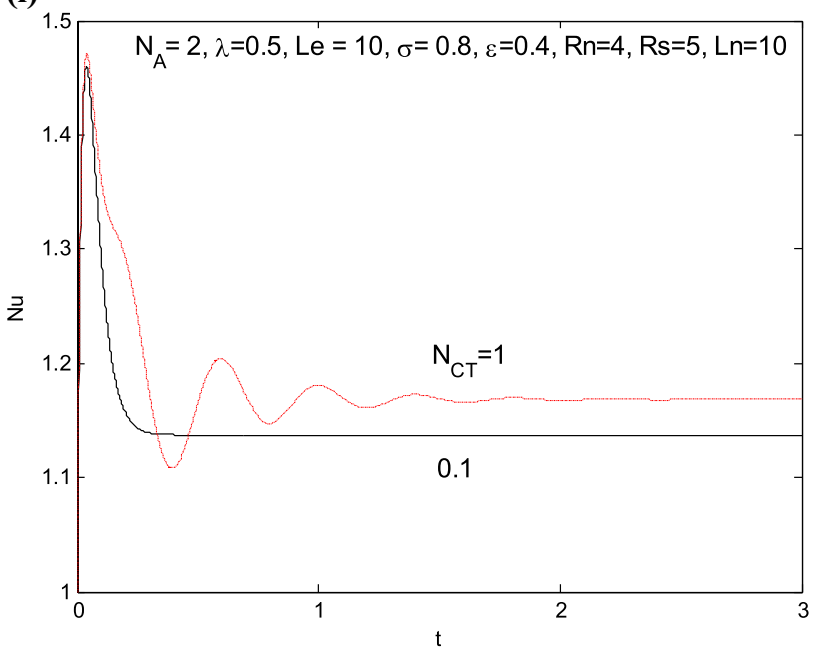

(h)

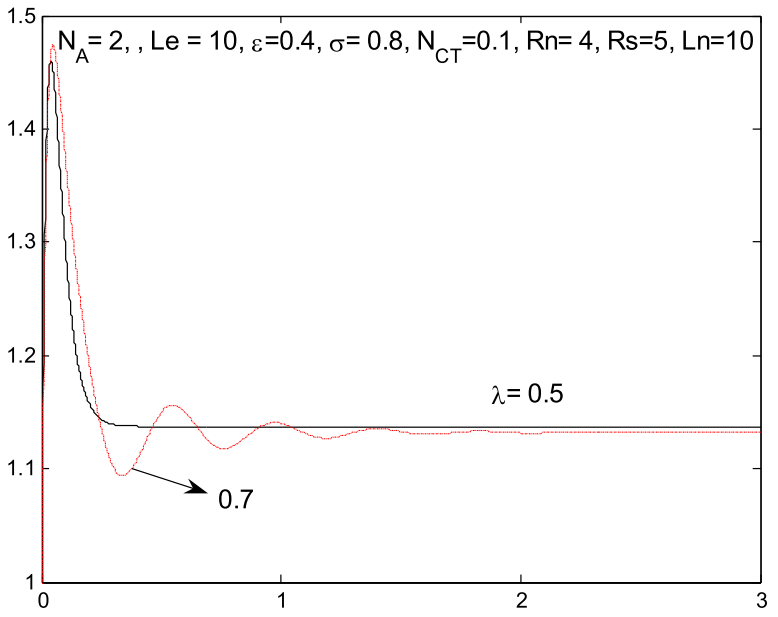

Fig. 12 (continued) 
(a)

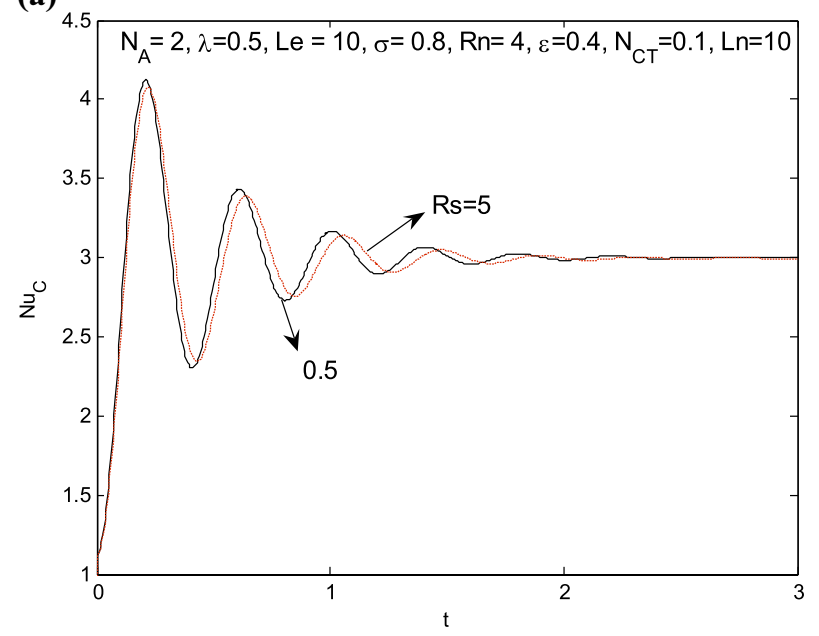

(c)

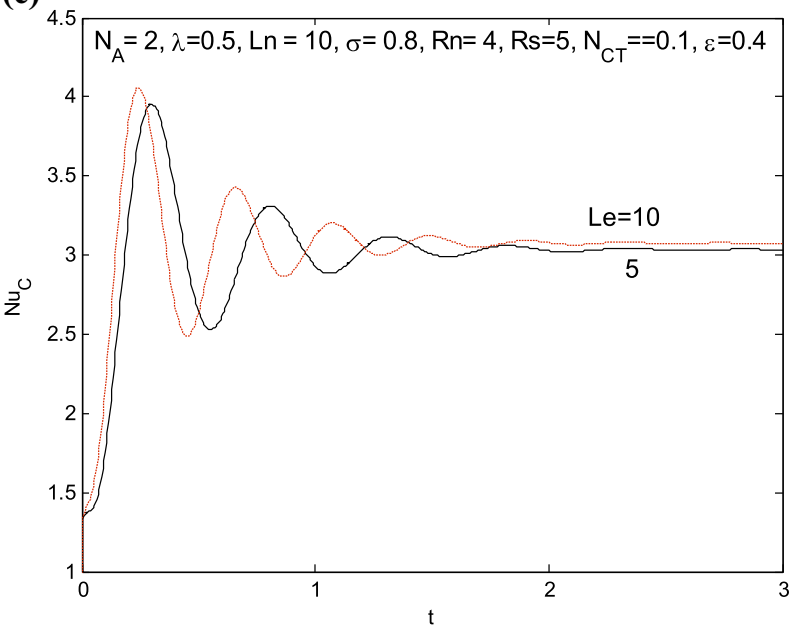

(e)

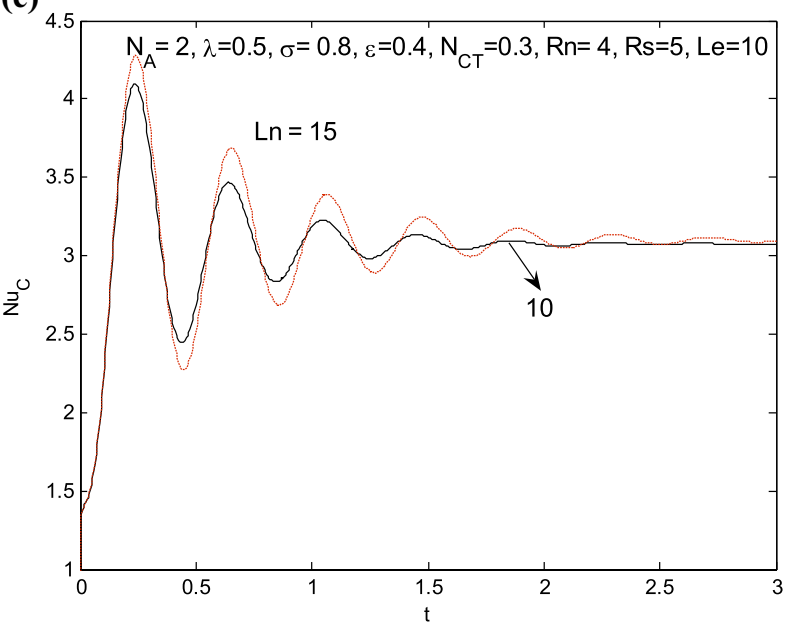

(b)

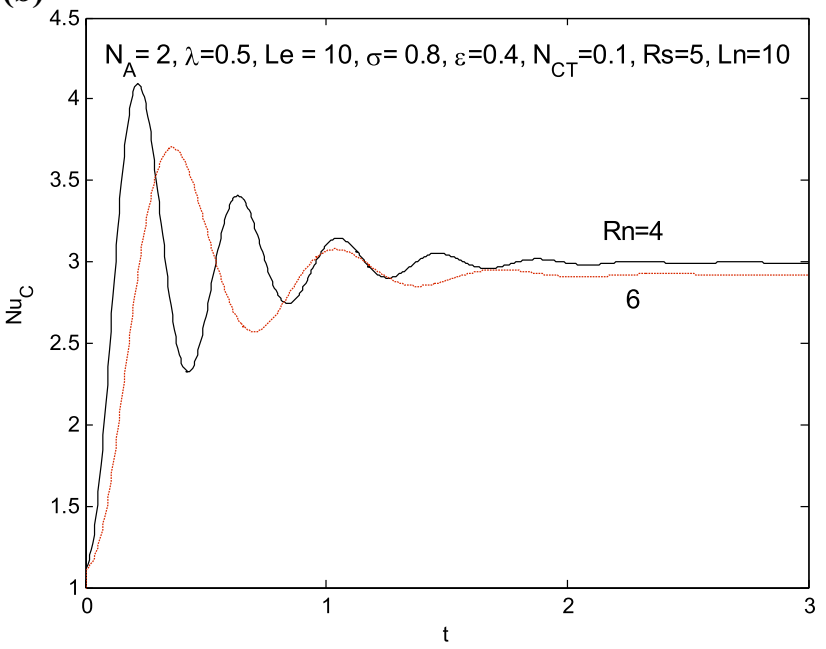

(d)

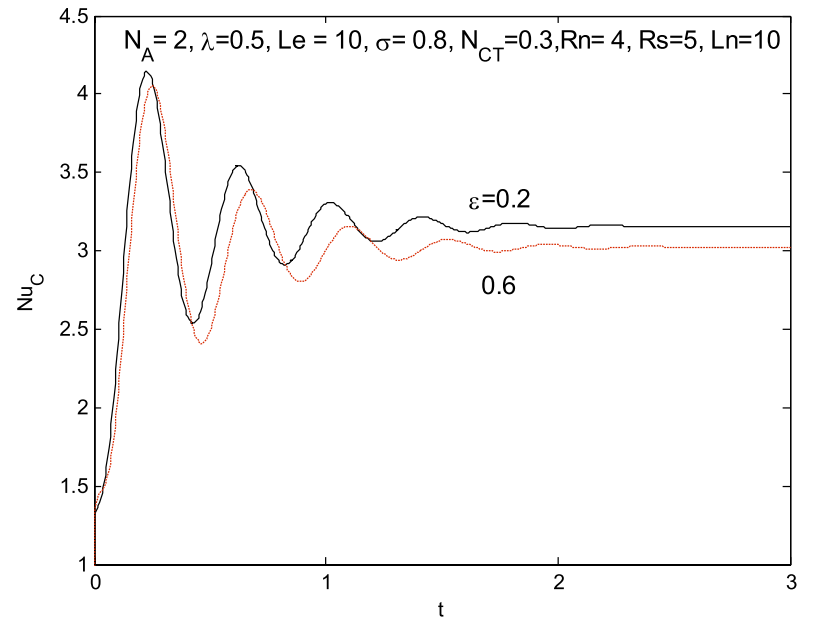

(f)

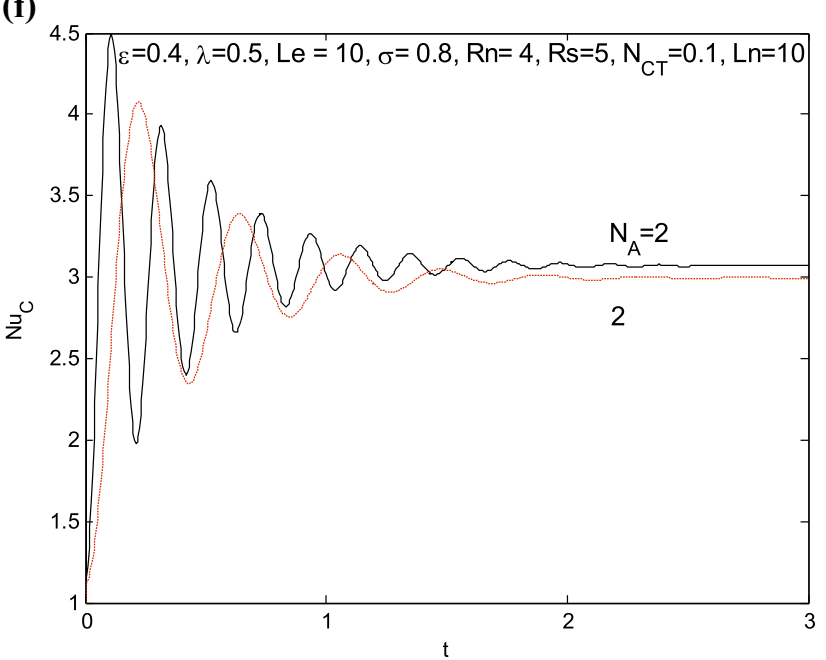

Fig. $13 N u_{C}$ versus $t$ for a $R s$ b $R n$ c Le d $\varepsilon$ e $L e f N_{A} \mathbf{g} \sigma \mathbf{h} \lambda \mathbf{i} N_{C T}$ 
(g)

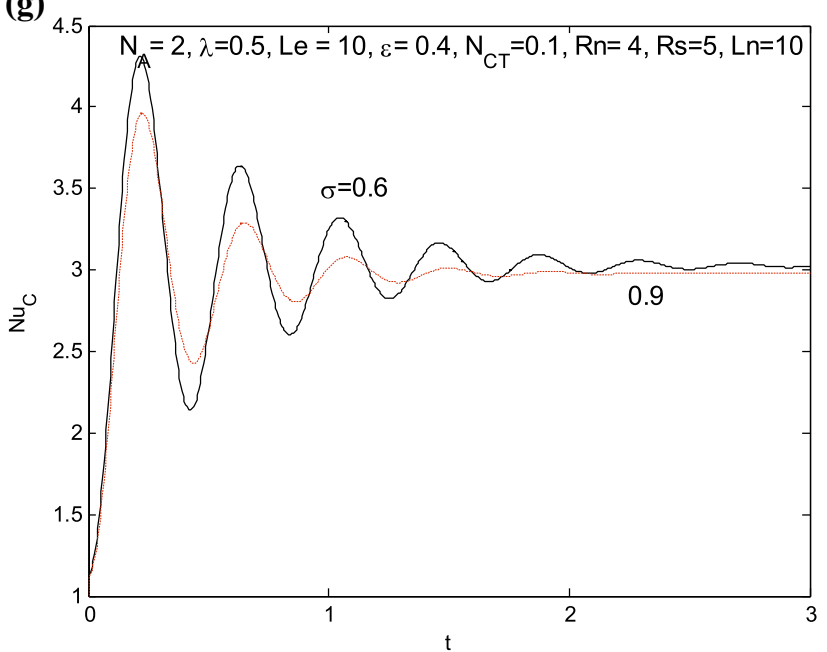

(i)

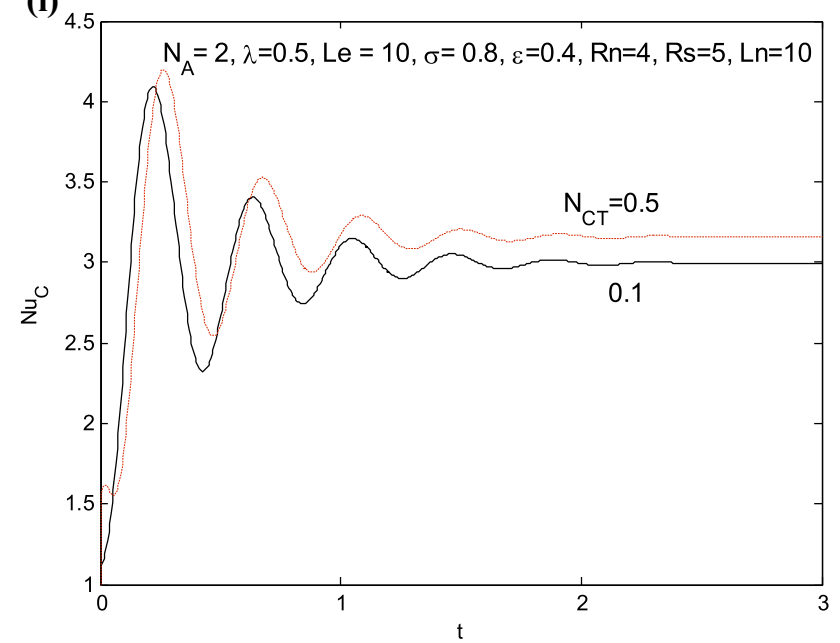

(h)

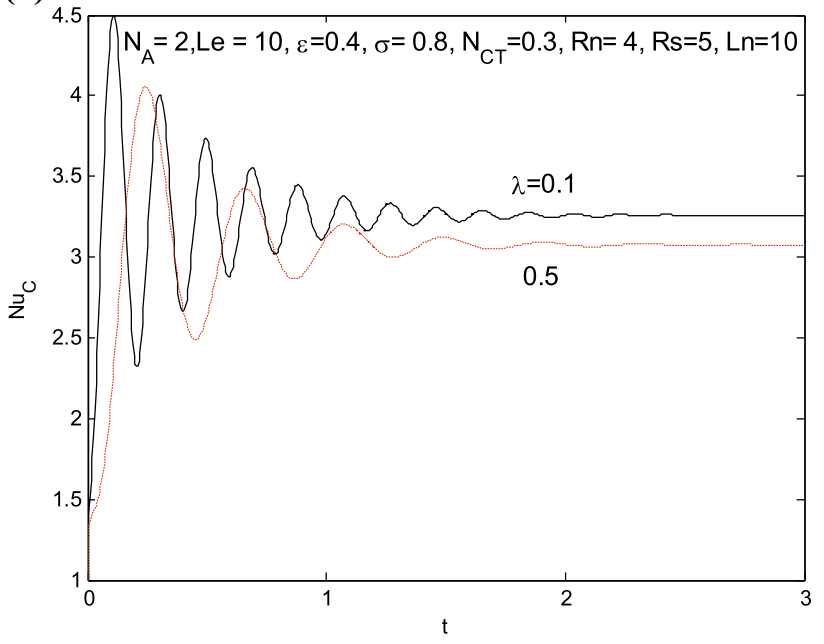

Fig. 13 (continued) 
(a)

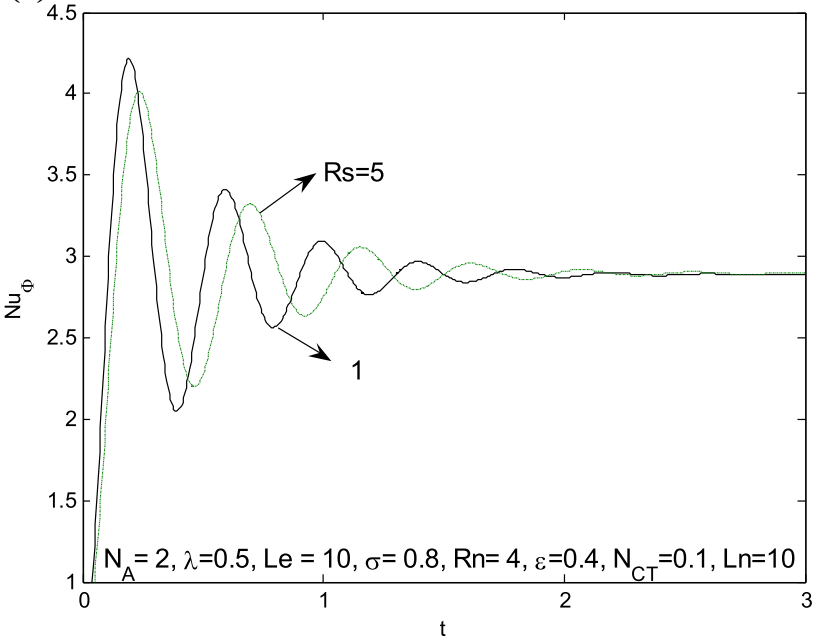

(c)

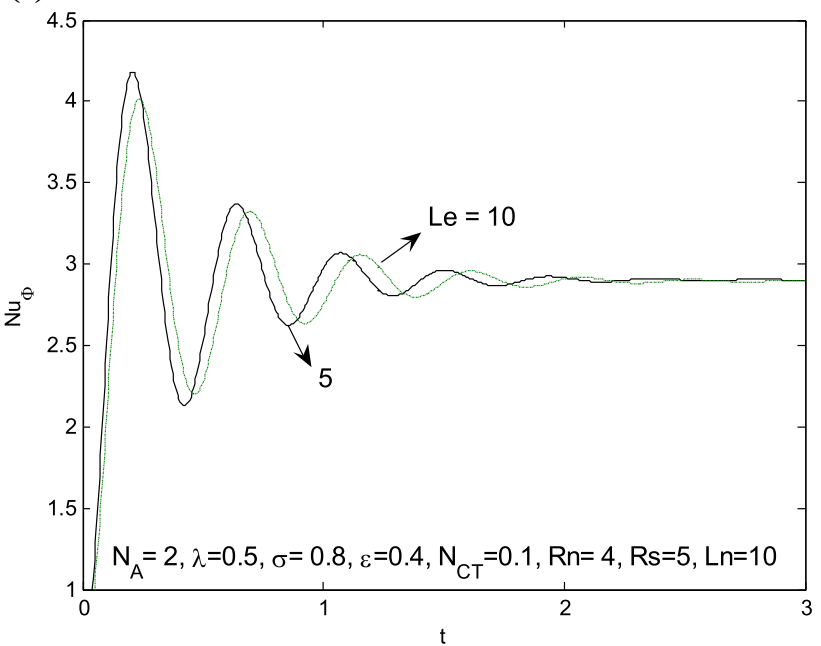

(e)

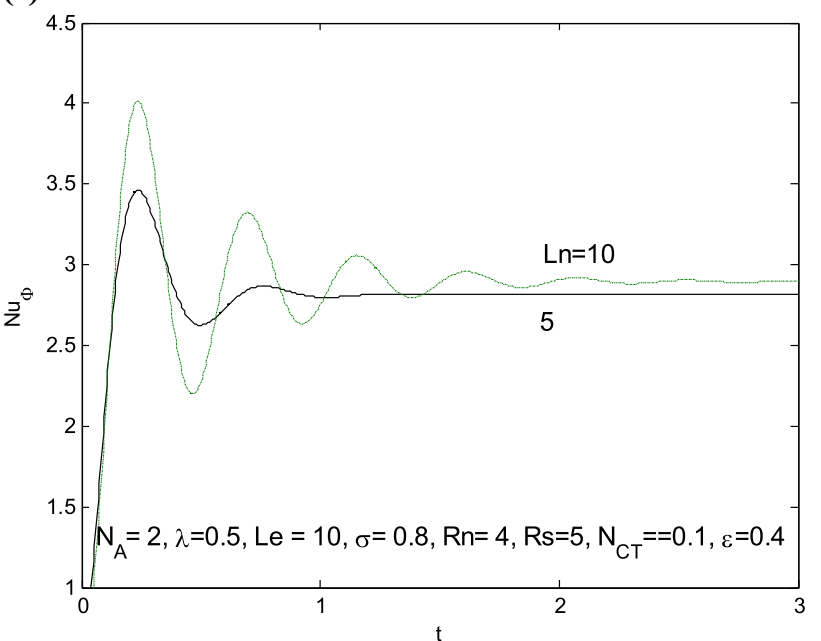

(b)

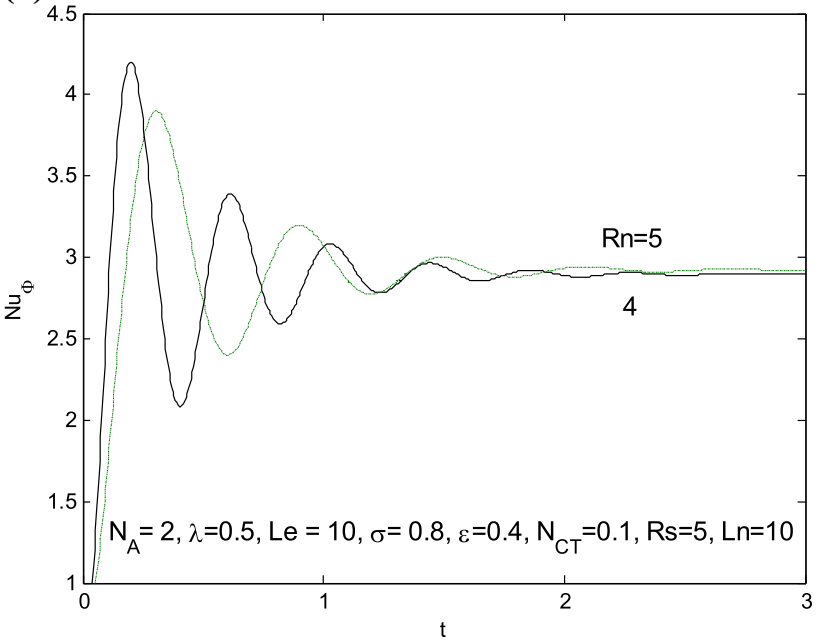

(d)

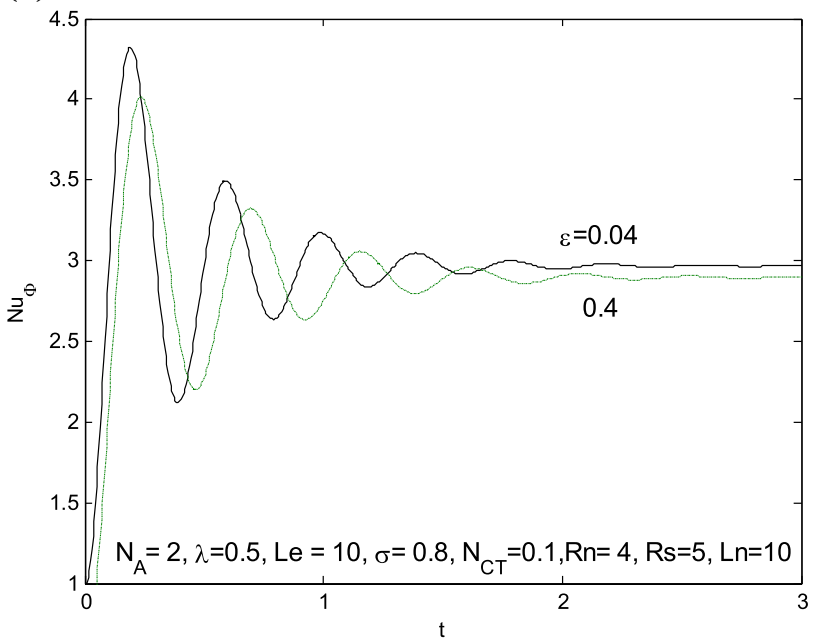

(f)

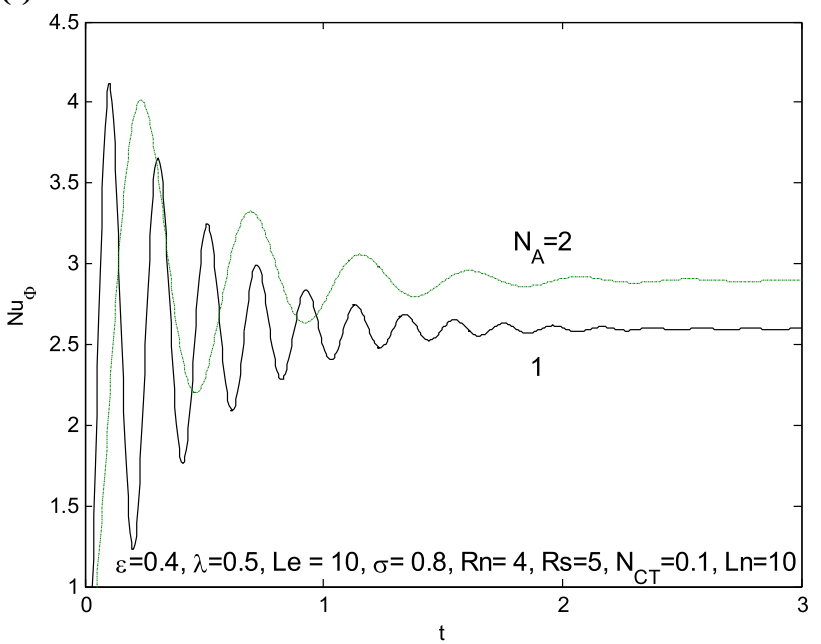

Fig. $14 N u_{\phi}$ versus $t$ for a $R s \mathbf{b} R n \mathbf{c} L e \mathbf{d} \varepsilon$ e $L n \mathbf{f} N_{A} \mathbf{g} \sigma \mathbf{h} \lambda \mathbf{i} N_{C T}$ 
(g)

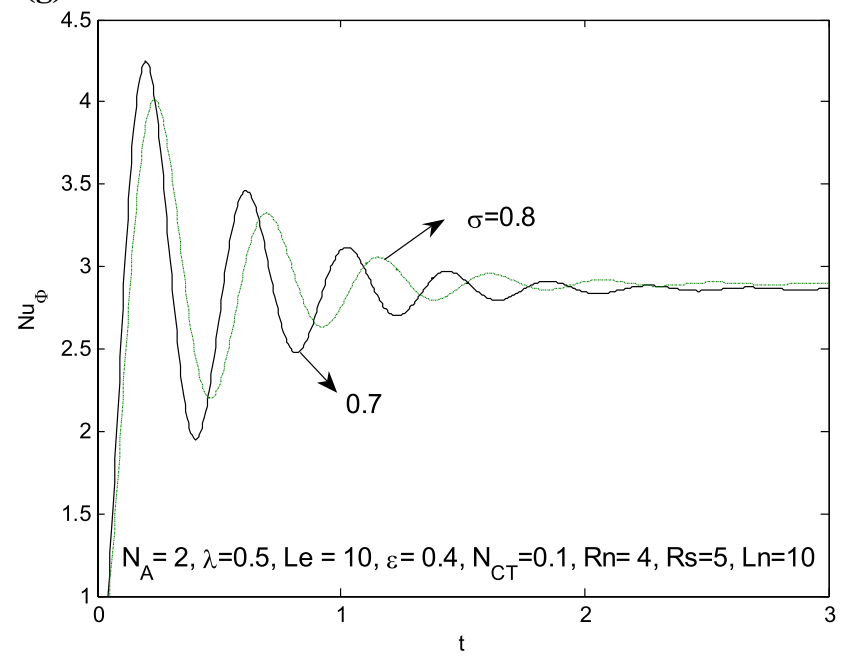

(i)

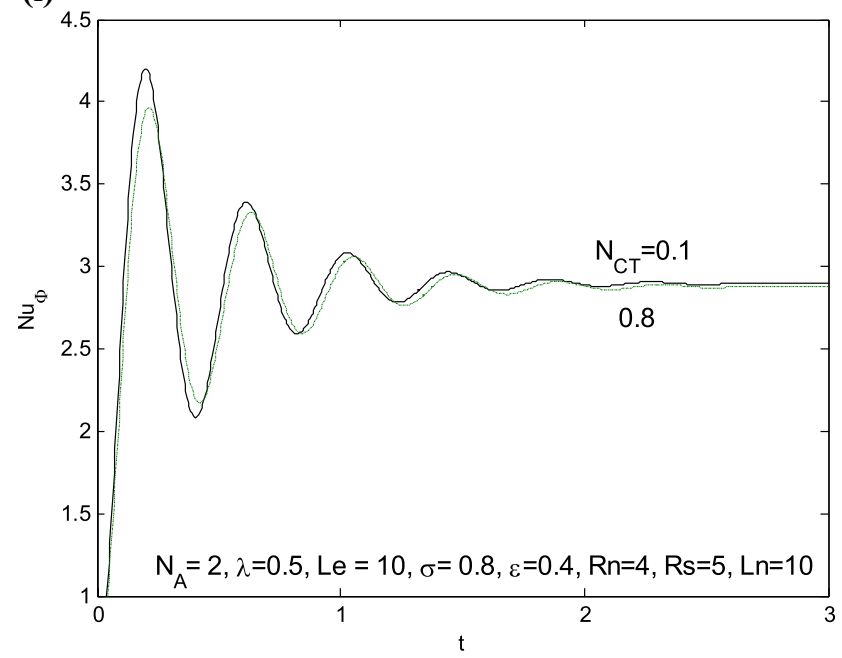

(h)

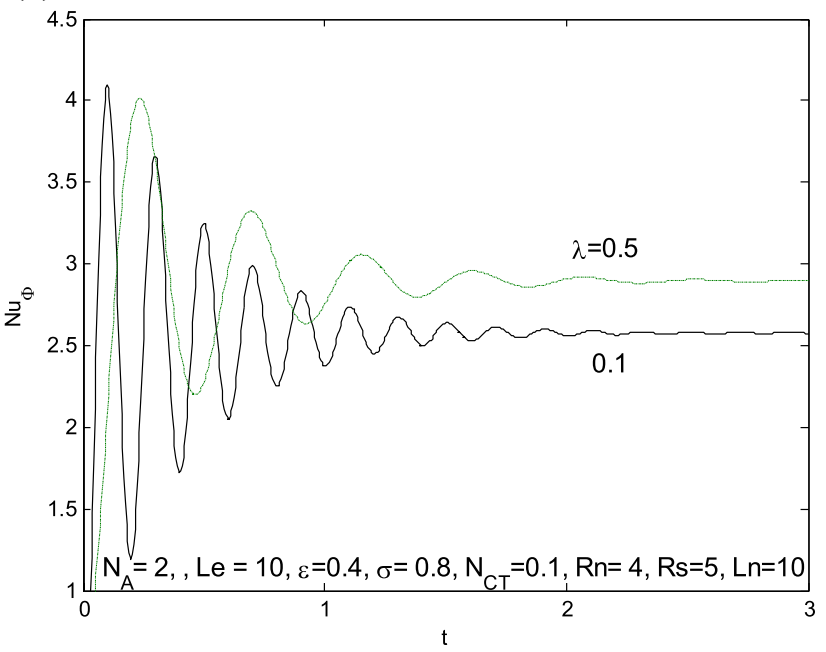

Fig. 14 (continued) 


$$
t=0.02
$$

(a)

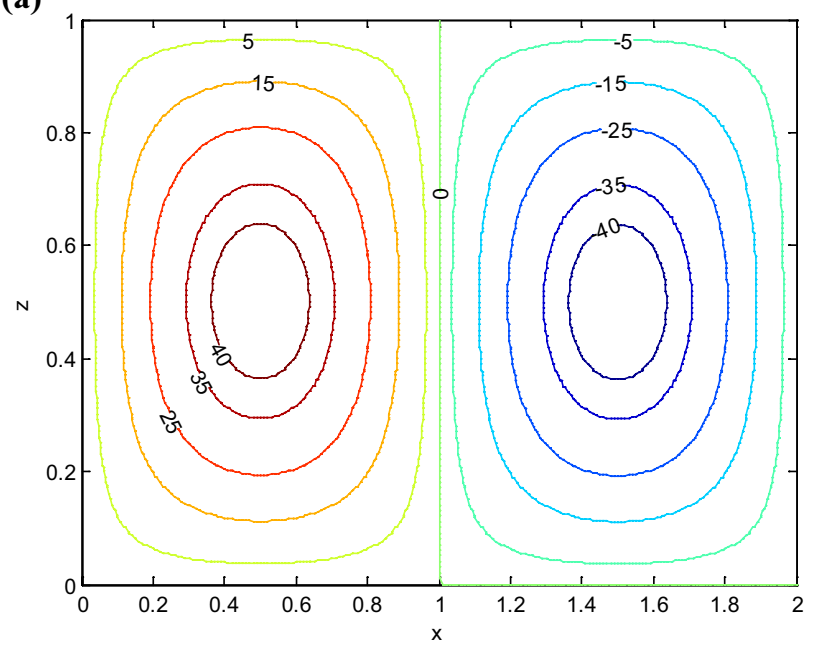

(c)

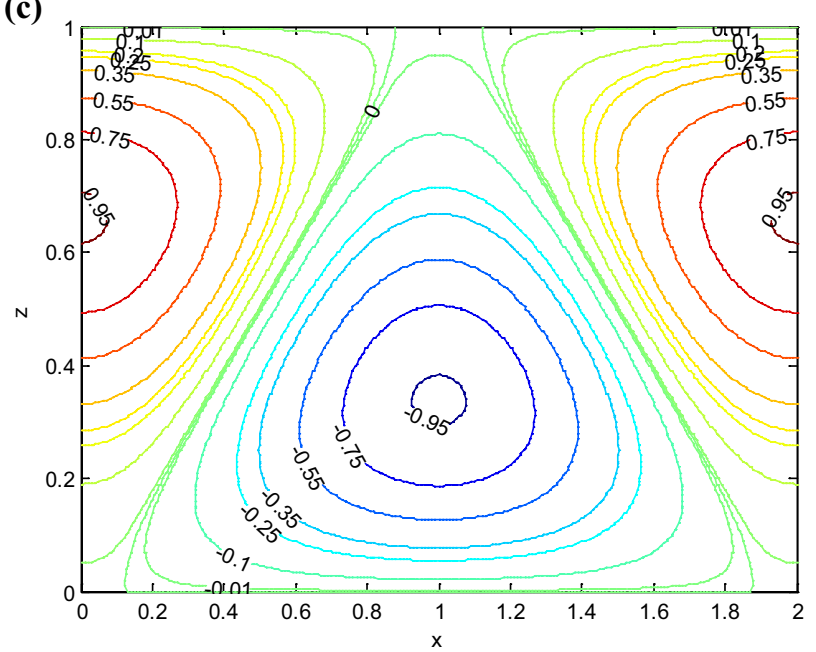

(e)

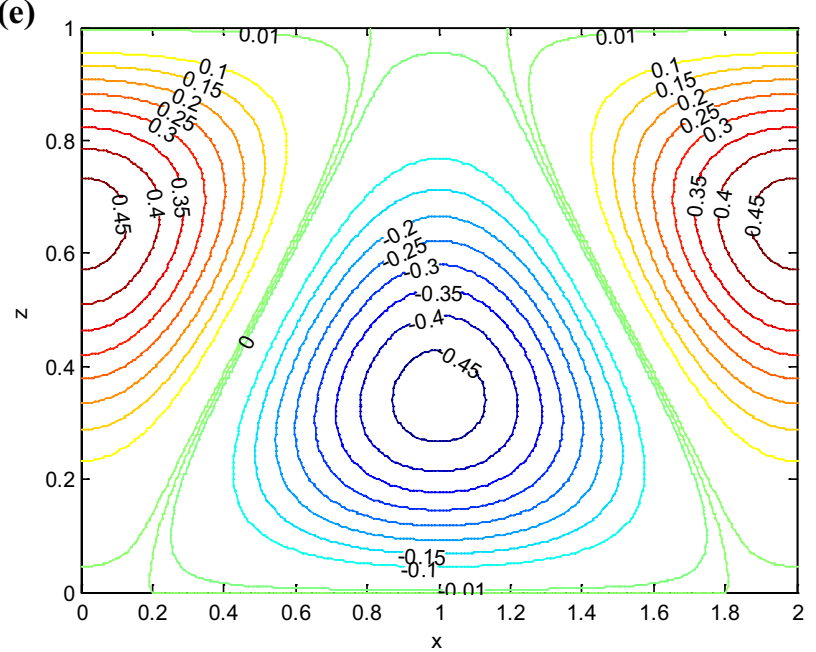

$t=2$

(b)

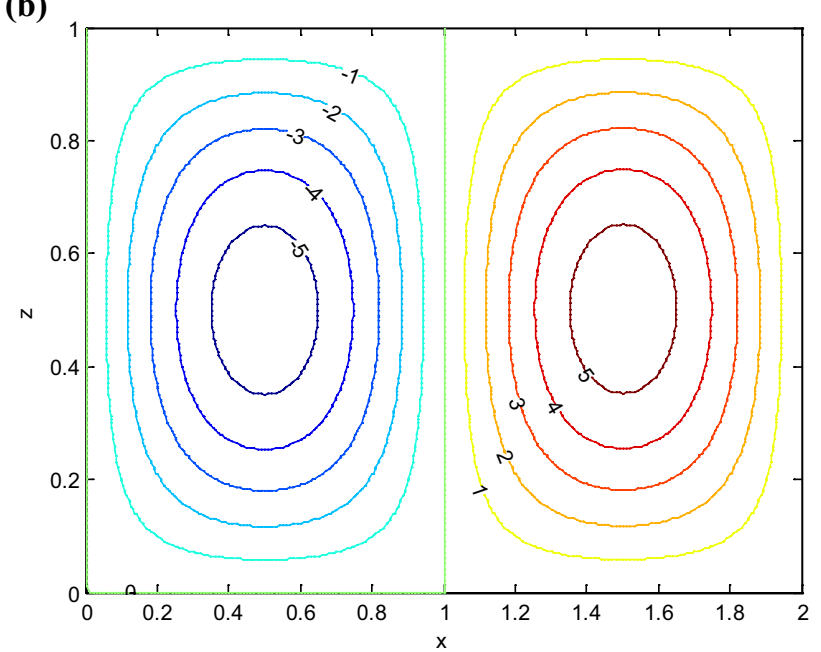

(d)

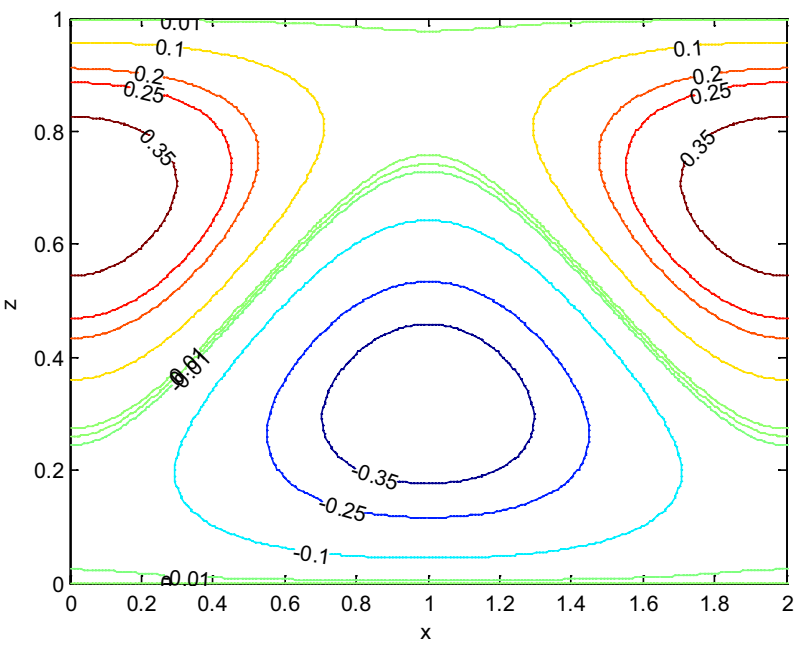

(f)

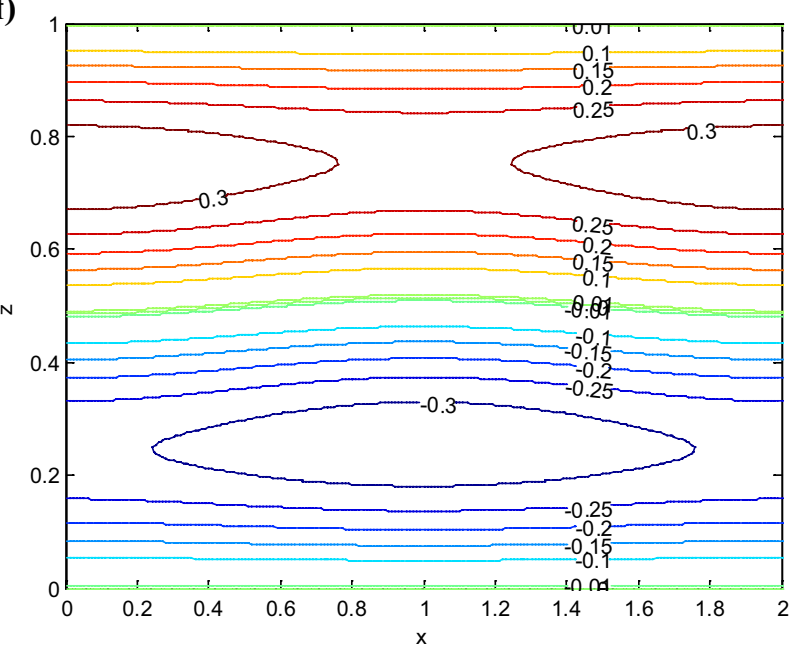

Fig. 15 Streamlines, isotherms, isonanoconcentration, isohalines for $R s=2, R n=0.5, L e=10, \varepsilon=0.4, L n=5, N_{A}=2, \sigma=0.5, \lambda=0.1, N_{C T}=0.1$ 
(g)

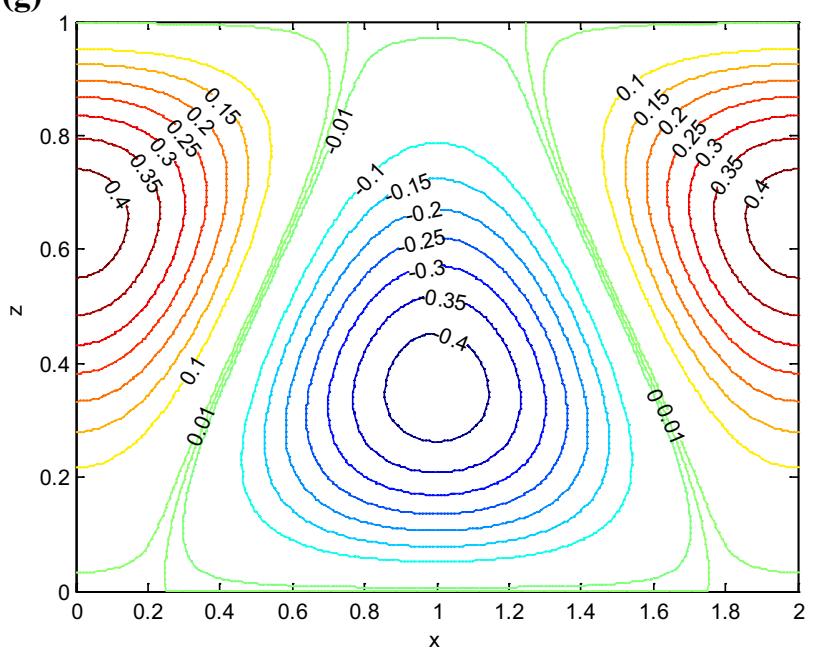

(h)

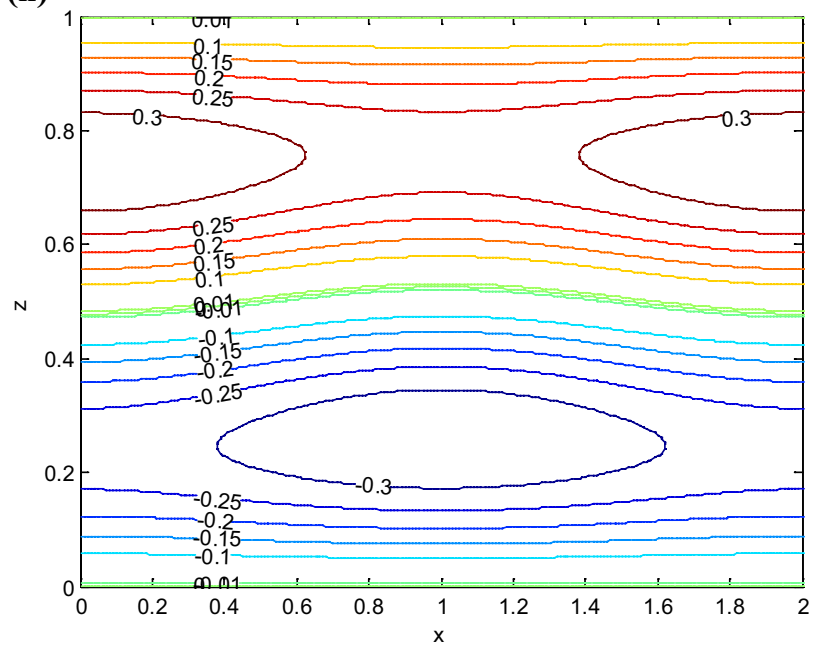

Fig. 15 (continued)

Acknowledgements The authors wish to gratefully acknowledge financial support through major research Project No. F. 42-9/2013 (SR) from the University Grants Commission (UGC), New Delhi.

\section{Compliance with ethical standards}

Conflict of interest The authors declare that they have no conflict of interest.

\section{References}

1. Khuzhayorov B, Auriault JL, Royer P (2000) Derivation macroscopic filtration law for transient linear fluid flow in porous media. Int J Eng Sci 38:487-504

2. Soret Ch (1880) Influence de la température sur la distribution des sels dans leurs solutions. Acad Sci Paris 91:289-291

3. Costesèque $P$, Fargue $D$, Jamet $P(2002)$ Thermodiffusion in porous media and its consequences. In: Thermal nonequilibrium phenomena in fluid mixtures (lecture notes in physics), vol 584. Springer, Berlin, pp 389-427

4. Ludwig C (1881) Diffusion zwischen ungleich erwwärmten orten gleich zusammengestzter lösungen. Sitz. Ber Akad Wiss Wien Math-Naturw KI 20:539

5. De Groot SR, Mazur P (1962) Non-equilibrium thermodynamics. North-Holland Publishing Company, Amsterdam

6. Tyrrell CJ (1965) The electrodeposition of iridium. Trans Inst Metal Finish 43:161-166

7. La PA, Surko CM (1998) Convective instability in a fluid mixture heated from above. Phys Rev Lett 80:3759-3762

8. Köhler W, Wiegand S (2008) Thermal nonequilibrium phenomena in fluid mixtures. Springer, Berlin

9. Platten JK, Chavepeyer G (1973) Oscillatory motion in Bénard cell due to the Soret effect. J Fluid Mech 60:305-319

10. Hardin GR, Sani RL, Henry D, Roux B (1990) Buoyancy-driven instability in a vertical cylinder: binary fluids with Soret effect. Part I: general theory and stationary stability results. Int J Numer Methods Fluids 10:79-117
11. Bourich M, Hasnaoui M (2004) Soret effect inducing subcritical and Hopf bifurcations in a shallow enclosure filled with a clear binary fluid or a saturated porous medium: a comparative study. Phys Fluids 16:551

12. Rahman MA, Saghir MZ (2014) Thermodiffusion or Soret effect: historical review. Int J Heat Mass Transf 73:693-705

13. Weinberger W (1964) The physics of the solar pond. Sol Energy 8:45-56

14. Gregg M (1973) The microstructure of the ocean. Sci Am 228:65-77

15. Spiegel EA (1972) Convection in stars-II: special effects. Annu Rev Astron Astrophys 10:261-304

16. Montel F (1994) Importance de la thermodiffusion en exploitation et production pétrolières. Entropie 83:184-185

17. Montel F (1998) La place de la thermodynamique dans une modélisation des répartitions des espéces d'hydrocarbures dans les réservoirs pétroliers: incidence sur les problémes de production. Entropie 214:7-10

18. Dominguez G, Wilkins G, Thiemens MH (2011) The Soret effect and isotopic fractionation in high-temperature silicate melts. Nature 473:70-73

19. Bonner FJ, Sundelöf LO (1984) Thermal diffusion as a mechanism for biological transport. Z Naturforschung C 310:656-661

20. Atia L, Givli S (2014) A theoretical study of biological membrane response to temperature gradients at the single-cell level. J R Soc Interface 11:20131207

21. Nazaroff WW, Cass GR (1987) Particle deposition from a natural convection flow onto a vertical isothermal flat plate. J Aerosol Sci 18:445-455

22. Derakhshan R, Ahmadreza S, Hosseinzadeha K, Nimafarb M, Ganji DD (2019) Hydrothermal analysis of magneto hydrodynamic nanofluid flow between two parallel by AGM. Case Stud Therm Eng 14:100439

23. Zangooee MR, Hosseinzadeh K, Ganji DD (2019) Hydrothermal analysis of MHD nanofluid ( $\mathrm{TiO}_{2}-\mathrm{GO}$ ) flow between two radiative stretchable rotating disks using AGM. Case Stud Therm Eng 14:100460

24. Ghadikolaei SS, Hosseinzadeh K, Ganji DD, Hatami M (2019) Investigation on magneto Eyring-Powell nanofluid flow over inclined stretching cylinder with nolinear thermal radiation and Joule heating effect. World J Eng 16(1):51-63 
25. Ghadikolaei SS, Hosseinzadeh K, Hatami M, Ganji DD, Armin $M(2018)$ Investigation for squeezing flow of ethylene glycol $\left(\mathrm{C}_{2} \mathrm{H}_{6} \mathrm{O}_{2}\right)$ carbon nanotubes (CNTs) in rotating stretching channel with nonlinear thermal radiation. J Mol Liq 263:10-21

26. Ghadikolaei SS, Hosseinzadeh K, Ganji DD, Jafari B (2018) Nonlinear thermal radiation effect on magneto Casson nanofluid flow with Joule heating effect over an inclined porous stretching sheet. Case Stud Therm Eng 12:176-187

27. Ghadikolaei SS, Hosseinzadeh K, Ganji DD, Hatami M (2018) $\mathrm{Fe}_{3} \mathrm{O}_{4}-\left(\mathrm{CH}_{2} \mathrm{OH}\right)_{2}$ nanofluid analysis in a porous medium under MHD radiative boundary layer and molecular dusty fluid. $\mathrm{J}$ Mol Liq 258:172-185

28. Gholinia M, Gholinia S, Hosseinzadeha K, Ganji DD (2018) Investigation on ethylene glycol nano fluid flow over a vertical permeable circular cylinder under effect of magnetic field. Results Phys 9:1525-1533

29. Hosseinzadeh K, Afsharpanah F, Zamani S, Gholinia M, Ganji DD (2018) A numerical investigation on ethylene glycol-titanium dioxide nanofluid convective flow over a stretching sheet in presence of heat generation/absorption. Case Stud Therm Eng 12:228-236

30. Hosseinzadeh K, Jafarian Amiri A, Ardahaie SS, Ganji DD (2017) Effect of variable Lorentz forces on nanofluid flow in movable parallel plates utilizing analytical method. Case Stud Therm Eng 10:595-610

31. Alishayev MG (1974) Proceedings of Moscow pedagogy institute. Hydromechanics 3:166-174

32. Wang S, Tan W (2011) Stability analysis of Soret-driven doublediffusive convection of Maxwell fluid in a porous medium. Int J Heat Fluid Flow 32:88-104

33. Tan WC, Masuoka T (2007) Stability analysis of a Maxwell fluid in a porous medium heated from below. Phys Lett $A$ 360:454-460

34. Wang S, Tan W (2008) Stability analysis of double-diffusive convection of Maxwell fluid in a porous medium heated from below. Phys Lett A 372:3046-3050

35. Awad FG, Sibanda P, Motsa Sandile S (2010) On the linear stability analysis of a Maxwell fluid with double-diffusive convection. Appl Math Model 34:3509-3517

36. Jaimala, Goyal N (2012) Soret Dufour driven thermosolutal instability of Darcy-Maxwell fluid. IJE Trans A Basics 25:367-377
37. Yang Z, Wang S, Zhao M, Li S, Zhang Q (2013) The onset of double diffusive convection in a viscoelastic fluid-saturated porous layer with non-equilibrium model. PLoS One 8:e7101056

38. Gaikwad SN, Kamble SS (2015) Theoretical study of cross diffusion effects on convective instability of Maxwell fluid in porous medium. Am J Heat Mass Transf 2:108-126

39. Jaimala, Singh R, Tyagi VK (2017) A macroscopic filtration model for natural convection in a Darcy Maxwell nanofluid saturated porous layer with no nanoparticle flux at the boundary. Int J Heat Mass Transf 111:451-466

40. Baehr HD, Stephan K (2011) Heat and mass transfer, 3rd edn. Springer, New York

41. Nield DA, Kuznetsov AV (2014) Thermal instability in a porous medium layer saturated by a nanofluid: a revised model. Int J Heat Mass Transf 68:211-214

42. Horton W, Rogers FT Jr (1945) Convection currents in a porous medium. J Appl Phys 16:367-370

43. Eastman A, Phillpot SR, Choi US, Keblinski P (2004) Thermal transport in nanofluids. Annu Rev Mater Res 34:219-246

44. Buongiorno J (2006) Convective transport in nanofluids. ASME $J$ Heat Transf 128:240-250

45. Nield DA, Kuznetsov AV (2010) Thermal instability in a porous medium layer saturated by a nanofluid. Int J Heat Mass Transf 52:5796-5801

46. Hurle DTJ, Jakeman E, Pike E (1967) On the solution of the Bénard problem with boundaries of finite conductivity. Proc $R$ Soc A 296:469-475

47. Chandrasekhar S (1981) Hydrodynamic and hydromagnetic stability. Dover Publications, New York

48. Jaimala, Singh R, Tyagi VK (2018) Stability of a double diffusive convection in a Darcy porous layer saturated with Maxwell nanofluid under macroscopic filtration law: a realistic approach. Int J Heat Mass Transf 125:290-309

49. Agarwal S, Rana P, Bhadauria BS (2014) Rayleigh-Bénard convection in a nanofluid layer using a thermal nonequilibrium model. J Heat Transf 136:122501

Publisher's Note Springer Nature remains neutral with regard to jurisdictional claims in published maps and institutional affiliations. 